\title{
Surface-based tracking for short association fibre tractography
}

\author{
Dmitri Shastina,b,c, $]$, Sila Genc ${ }^{a}$, Greg D. Parkera, Kristin Kollera, Chantal \\ M.W. Tax ${ }^{\mathrm{a}, \mathrm{d}}$, John Evans ${ }^{\mathrm{a}}$, Khalid Hamandia,c,e, William P. Gray, ${ }^{\mathrm{a}, \mathrm{b}, \mathrm{c}}$, Derek K. \\ Jones $^{\mathrm{a}, \mathrm{c}}$, Maxime Chamberland ${ }^{\mathrm{a}, \mathrm{f}}$ \\ ${ }^{a}$ Cardiff University Brain Research Imaging Centre (CUBRIC), Cardiff \\ University, Cardiff, United Kingdom \\ ${ }^{b}$ Department of Neurosurgery, University Hospital of Wales, Cardiff, United Kingdom \\ ${ }^{c}$ BRAIN Biomedical Research Unit, Health \& Care Research Wales, Cardiff, United \\ Kingdom \\ dImage Sciences Institute, University Medical Center Utrecht, Utrecht, Netherlands \\ ${ }^{e}$ Department of Neurology, University Hospital of Wales, Cardiff, United Kingdom fDonders Institute for Brain, \\ Cognition and Behaviour, Radboud University, Nijmegen, Netherlands
}

\begin{abstract}
Short association fibres (SAF) of the human brain are estimated to represent over a half of the total white matter volume, and their involvement has been implicated in a range of neurological and psychiatric conditions. This population of fibres, however, remains relatively understudied in the neuroimaging literature. Some of the challenges pertinent to the mapping of SAF include their variable anatomical course and close proximity to the cortical mantle, leading to partial volume effects and exacerbating the influence of the gyral bias. This work considers the choice of scanner, acquisition, voxel size, seeding strategy and filtering techniques to propose a whole-brain, surface-based
\end{abstract}

\footnotetext{
${ }^{\square}$ Corresponding author. Address: CUBRIC, Maindy Rd, Cardiff, United Kingdom, CF24 $4 \mathrm{HQ}$

Email address: sastind@cardiff.ac.uk (Dmitri Shastin)
} 
tractography approach with the aim of providing a method for investigating SAF $\leq 30-40 \mathrm{~mm}$. The framework is designed to: (1) ensure a greater cortical surface coverage through spreading streamline seeds more uniformly; (2) introduce precise filtering mechanics which are particularly important when dealing with small, morphologically diverse structures; and (3) allow the use of surface-based registration for dataset comparisons which can be superior to volume-based registration in the cortical vicinity. The indexation of surface vertices at each streamline end enables direct interfacing between streamlines and the cortical surface without dependence on the voxel grid. SAF tractograms generated using recent testretest data from our institution are carefully characterised and measures of consistency using streamline-, voxel- and surface-wise comparisons calculated to inform researchers and serve as a benchmark for future methodological developments.

Keywords: Short association fibers, U-fibers, superficial white matter, tractography, surface, consistency

\section{Introduction}

Functional integration of the brain subunits is mediated in part by the white matter (Neubert et al. (2010)), which comprises a vast network of connections between neuronal populations and has been shown to exhibit change in response to physiological processes (Scholz et al. (2009); Hihara et al. (2006); Dubois et al. (2014); de Groot et al. (2015); Slater et al. (2019)) and disease (Mito et al. (2018); Datta et al. (2017); de Schipper et al. (2019)). The white matter is typically divided into projection, 
commissural and association fibres. It is estimated that the association fibres dominate the white matter (Schüz and Braitenberg (2002)), connecting the cortical areas within hemispheres. They are in turn subdivided into long and short range (local) fibre, sometimes also distinguishing neighbourhood association fibres (Schmahmann and Pandya (2006)). The long-range fibres course in the depth of the white matter, connecting distant areas of the hemisphere and forming distinct bundles that have largely consistent anatomy across individuals. Conversely, the short association fibres (SAF) connect adjacent cortical areas. Their most superficial component is often referred to as the U-shaped fibres and described as a thin band that runs immediately beneath the sixth layer of the cortex (Schmahmann and Pandya (2006)) encompassing a single gyrus or sulcus (Schüz and Braitenberg (2002)). It is established that neighbouring cortical areas exhibit the strongest structural connectivity (Markov et al. (2014)). Further, it is estimated that only ?10\% of the cortico-cortical connections belong to the long fascicles, with the volume of the U-shaped fibres possibly as much as $260 \%$ of the total white matter volume (Schüz and Braitenberg (2002)). It is remarkable therefore that in the neuroimaging literature SAF have only started to gain more attention recently (Ouyang et al. (2017)).

\begin{tabular}{ll}
\hline Abbreviation & Meaning/Interpretation \\
\hline ACT & Anatomically-constrained tractography \\
CVB & Between-subject coefficient of variation \\
CVW & Within-subject coefficient of variation \\
dMRI & Diffusion magnetic resonance imaging \\
DSI & Diffusion spectrum imaging
\end{tabular}




\begin{tabular}{ll} 
DTI & Diffusion tensor imaging \\
FA & Fractional anisotropy \\
fODF & Fibre orientation distribution function \\
FWHM & Full-width half-maximum \\
GG & Grey-grey filter \\
GMWMI & Grey matter - white matter interface \\
GWG & Grey-white-grey filter \\
HARDI & High angular resolution diffusion imaging \\
HH & Hemisphere-hemisphere filter \\
ICC & Intraclass correlation coefficient \\
LCHT & Local cortical half-thickness \\
MCC & Mid-cortical coordinate \\
PSM & Pial surface mesh \\
SA & "State-of-the-art" acquisition \\
SAF & Short association fibres \\
ST & "Standard" acquisition \\
TDI & Track density imaging \\
WSM & White surface mesh \\
\hline &
\end{tabular}

Table 1: Table of abbreviations

Diffusion MRI (dMRI) is the preferred method for studying structural properties and connectivity of white matter pathways in vivo. Its sensitivity to the random microscopic motion of water molecules (Stejskal and Tanner (1965)) enables judgement to be made regarding the local directional architecture and microstructural properties (Pierpaoli et al. (1996)) of the fibers.

In the past few years, a number of dMRI-based studies have shown that SAF are affected by age and sex (Phillips et al. (2013)) as well as pathology including autism (d'Albis et al. (2018)), schizophrenia (Phillips et al. (2011), 
encephalitis (Phillips et al. (2018)) and epilepsy (0'Halloran et al. (2017); Liu et al. (2016); Govindan et al. (2013)), among others. dMRI methods used to study SAF can be broadly divided into those that do not use tractography and those that do. The former typically sample measures of microstructure in the superficial white matter as defined by regions of interest (Nazeri et al. (2013)) or uniformly along the cortical surface (Phillips et al. (2013, 2018, 2011); Liu et al. (2016)). This approach avoids any biases of tractography and can be less affected by the differences in cortical folding through the use of surface registration (Fischl et al. (1999)) but it does not discriminate between SAF and the superficial component of the long-range connections. On the other hand, tractography-based methods capitalise on local fibre orientation modelling which allows reconstruction of streamlines providing information about white matter morphology (Mori and Van Zijl (2002)). Numerous challenges, such as the inability to resolve multiple fibre directions in regions with complex fibre configurations, can create ambiguity and lead to a high number of false positive and false negative results during streamline generation (Maier-Hein et al. (2017)). Recent advances in image acquisition (Jones et al. (2018)) and processing as well as development of advanced fibre orientation estimation (Tournier et al. (2007); Dhollander et al. (2016); Jeurissen et al. (2014)) and streamline integration and filtering algorithms (Smith et al. (2012); Daducci et al. (2015); Smith et al. (2015)) have improved the quality of tractography. Despite this, available tools are typically used to study whole-brain tractograms or focus on the deep white matter bundles that show consistent organisation across individuals and thus the performance of these tools for investigating SAF remains uncertain. 


\section{Challenges in SAF reconstruction}

\subsection{Tractogram generation}

The study of SAF is confounded by a number of anatomical considerations and methodological limitations (for an overview, see Guevara et al. (2020); Jeurissen et al. (2017); Rheault et al. (2020); Reveley et al. (2015)) which span initial tractogram generation, SAF-specific filtering and analysis. The tractogram generation step faces the challenges of partial volume effects (due to the proximity of SAF to the cortex and CSF spaces) and complex local anatomy with multiple regions of crossing, bending, kissing, and fanning fibres. The subcortical location makes SAF potentially more sensitive to the so-called "gyral bias" - the phenomenon in tractography where many more streamlines terminate in the gyral crowns as opposed to the sulcal fundi (Li et al. (2010); Nie et al. (2011); Chen et al. (2012); Cottaar et al. (2021)). It remains unclear just how much of this effect is explained by methodological shortcomings (and can be improved) rather than underlying anatomy (see Van Essen et al. (2014) for a detailed discussion of the subject). SAF may in fact be promoting gyral bias by diverting tracking in the subcortical white matter in a tangential orientation (Reveley et al. (2015)). This tendency may prove beneficial for SAF tractography as tracking is encouraged along the natural SAF course. On the other hand, it is just as true for SAF as it is for tractography in general that sulcal fundi and/or gyral walls become harder to reach, affecting the distribution of streamlines on the cortical surface. The effect may be even more pronounced for SAF than the long tracts as the latter only adjoin the cortex in their periphery while SAF by definition may be affected throughout their full extent. Specifically in the context of Ushaped fibers, the 
gyral bias has been demonstrated even when state-of-the-art acquisition and tractography were used (Movahedian Attar et al. (2020)). It is therefore important to investigate strategies aimed at addressing this effect.

\subsection{Tractogram filtering}

From the filtering perspective, SAF may be defined locally based on manual dissections or functional MRI signal-derived cortical regions of interest (Movahedian Attar et al. (2020)), whilst for globally (brain-wise) defined SAF, the filtering criteria typically involve size, shape and/or cortical parcellation. Despite the existence of studies examining the histopathology of SAF in isolated brain regions, the absence of a detailed anatomical knowledge regarding the distribution and consistency of SAF on a whole-brain level or even a universally accepted definition (Ouyang et al. (2017)) complicates development and validation of non-invasive methods dedicated to the study of this subset of the white matter. For instance, the length definition of SAF (or U-shaped fibres) varies across sources. Some authors have focused on the relatively long streamlines of $20-80 \mathrm{~mm}$ (Guevara et al. (2017); Kai and Khan (2019)) or more (Román et al. (2017)), mainly concerning the bundles connecting neighbouring gyri; while others (Song et al. (2014); Movahedian Attar et al. (2020)) included the smaller range of 3-30 $\mathrm{mm}$ based on the classification by Schüz and Braitenberg (2002). Next, although using streamline similarity measures (typically shape and distance metrics) as filtering criteria (Román et al. (2017); 0'Halloran et al. (2017); Kai and Khan (2019)) may appear appealing, this may lead to exclusion of otherwise valid streamlines as SAF have been demonstrated to exhibit complex, diverse morphology (Movahedian Attar et 
al. (2020)) and varying spatial overlap (Zhang et al. (2010)); this is particularly true for shorter (<35 mm) streamlines (Román et al. (2017)). The use of cortical parcellations (division of the cortical mantle into discrete areas) can carry uncertainties of its own. The choice of parcellation scheme, termination criteria during tracking, and the way streamlines are associated with individual parcels all influence the result (Yeh et al. (2019)). Streamlines with both ends in the same parcel, particularly if larger parcels and discrete borders are used, can form the bulk of SAF tractograms and must not therefore be excluded from analysis.

\subsection{Tractogram comparison}

Group-wise analysis of SAF is challenged by inter-subject variations in cortical folding (Rademacher (2002)). Even the sulci known to exhibit more anatomical consistency across individuals (such as those corresponding to the primary somatosensory areas (Rademacher (2002)) demonstrate individual morphological differences up to $1-2 \mathrm{~cm}$ in a common reference frame (Steinmetz et al. (1989)). The trajectories of short (up to $40 \mathrm{~mm}$ ) superficial streamlines appear to be strongly influenced by the gyral pattern (Bajada et al. (2019)). Taken together, one should expect low consistency when comparing SAF tractograms composed of shorter streamlines between individuals based on their shape or spatial distribution alone. Connectome-based comparisons using cortical parcellations are possible yet again they face the same challenges as described above.

Our aim was to develop a methodological framework suitable for wholebrain tractography of SAF $\leq 30-40 \mathrm{~mm}$. We hypothesised that distributing streamline seeds more evenly on the cortical surface as well as 
employing surface-based filtering techniques may facilitate a more welldistributed final tractogram. We also aimed to investigate whether higher angular resolution, higher b-values and increased number of shells would make tracking more sensitive to the complex configuration of the subcortical white matter and enhance tissue type discrimination therefore better handling partial volume effects. To produce SAF-specific tractograms, we introduced simple, anatomy-driven filtering criteria that did not require manual dissection/pruning or the use of additional shape/parcellationbased priors. The tractograms were then assessed for within-subject consistency to evaluate the framework, coupled with between-subject analyses to inform the reader of what could be expected during the use of the method for population studies. It is also hoped that these data will facilitate ongoing methodological developments by acting as a source for benchmarking.

\section{Material and methods}

\subsection{Proposed surface-based SAF tractography framework}

The overall workflow is summarised in Figure 1. A FreeSurfer-generated fine cortical mesh (Fischl (2012)) is used to place streamline seeds in dMRI space. Tracking is performed using a probabilistic tracking algorithm with fibre orientation distribution functions (fODFs) generated from upsampled, multishell, high b-value DWI data. As this work focuses on short association fibres specifically, the maximum streamline length is set to $40 \mathrm{~mm}$.

By definition, a streamline representing association fibres must satisfy the following criteria: (1) both ends terminate in the neocortex; (2) both 
ends terminate in the same hemisphere; (3) the streamline courses through the white matter. Three respective filters ensuring these three criteria are met are applied to the initial tractogram. First, for each streamline end the closest point on the mid-cortical mesh is identified. The streamline terminates within the cortex if the distance to this closest point is smaller than half of the cortical thickness at that point. Second, a prior knowledge of which hemisphere each surface point belongs to ensures that the streamline starts and ends in the same hemisphere. Finally, the course within the white matter is confirmed by identifying two intersections with the white matter surface.

The following paragraphs detail each of the steps described.

\subsubsection{Streamline generation}

The FreeSurfer white matter surface mesh (WSM) typically contains ? 1.5 vertices $/ \mathrm{mm}^{2}$ for a total of ?250K vertices (points) for both hemispheres (excluding the medial wall) and an average face (triangle) area of $0.32 \mathrm{~mm}^{2}$ (range: $0.07-0.7 \mathrm{~mm}^{2}$, top and bottom $2 \%$ excluded) representing a reasonably dense and even spread; this can be further remeshed if needed. Vertex coordinates in each hemisphere are transformed to dMRI space with ANTs (Avants et al. (2009)) using the inverse warp (see subsubsection 3.2.1) and concatenated into a single array used to initiate seeding with MRtrix 3.0 (Tournier et al. (2019)). To this end, MRtrix was modified such that it could read coordinates from the array and use them as seeds with equal weights during tractogram generation. This seeding mechanism was verified by visual inspection of the seed distribution on T1weighted volumes co-registered to dMRI space and by comparing the input 
and output seed coordinates (data not shown). Next, tracking is performed using the second-order integration probabilistic algorithm iF0D2 (Tournier et al. (2010)) due to the expected large number of fibre crossings and challenging morphology; probabilistic tracking has previously been demonstrated to result in improved gyral bias (Nie et al. (2011)) and greater spatial overlap of SAF (Guevara et al. (2020)). The "seeds" parameter is set to 5 million to ensure an adequate number of streamlines per vertex. An additional restriction on the maximum streamline length of $40 \mathrm{~mm}$ is used to be consistent with the SAF definition of Schüz and Braitenberg (2002) and accounting for the fact that intracortical portions are later truncated (see section 3.1.2). Other parameters are left as their default settings in the MRtrix implementation of iFOD2 (max angle: $45^{\circ}$, step size: $0.5 \mathrm{~mm}$, threshold: 0.05 , FOD power: 0.25 ).

\subsubsection{Streamline filtering}

Grey-grey (GG) filter. To identify streamlines starting and ending in the neocortex, midcortical coordinates (MCC) are defined by averaging coordinates of the matching WSM and pial surface mesh (PSM) vertices. Next, local cortical half-thickness (LCHT) is defined as the Euclidean distance between the MCC and the corresponding WSM vertex to account for local variation in cortical thickness. Both ends of each streamline in the initial tractogram are then evaluated for "intracortical position" by (1) identifying the closest MCC, (2) measuring the Euclidean distance to it, (3) comparing this distance to the LCHT measure of said MCC. The "intracortical position" is confirmed if the streamline terminates in a sphere centred on the MCC and with the radius LCHT (also see Appendix B). 


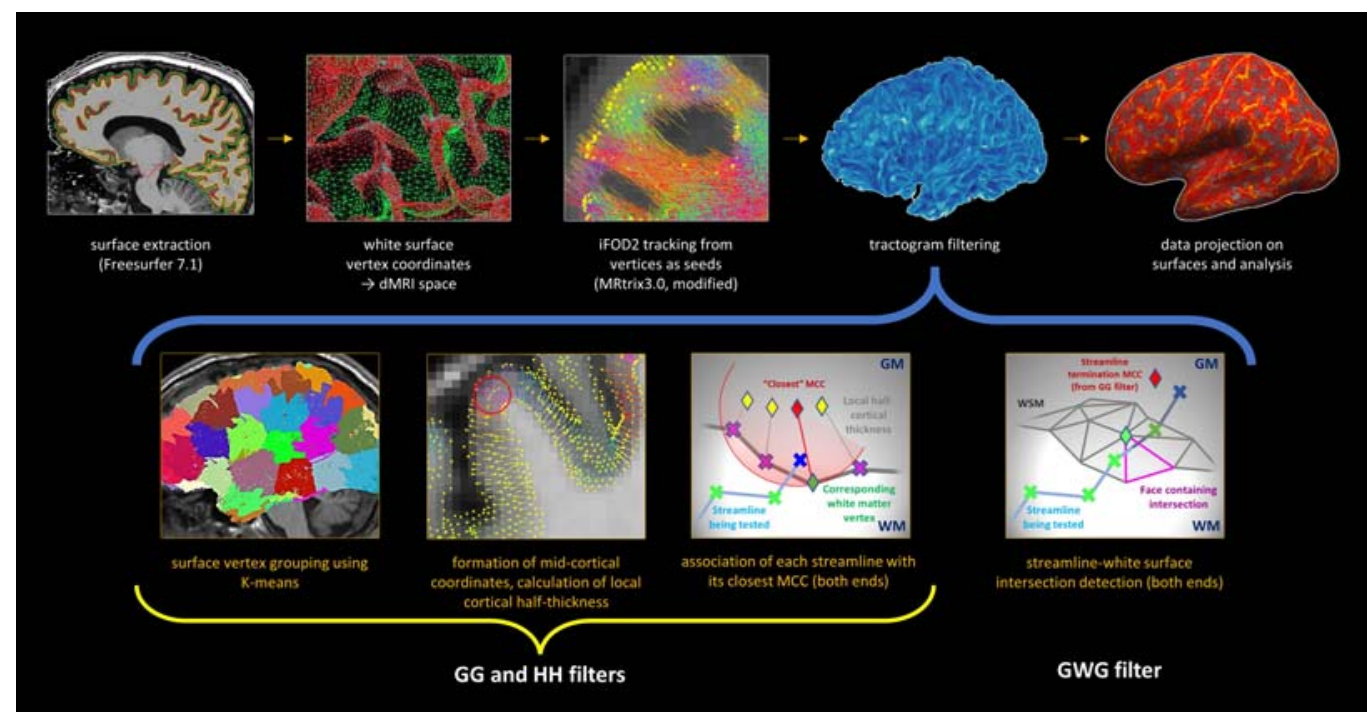

Figure 1: Pipeline summary. After seeding from white surface mesh (WSM) coordinates (top row, 1-3), tractograms are filtered (top row, 4) to ensure each streamline starts and ends in the neocortex (grey-grey filter) of the same hemisphere (hemisphere-hemisphere filter) and escapes into white matter along the way (grey-white-grey filter). The grey-grey filter (bottom row, 1-3) functions by finding the closest midcortical coordinate (MCC, average of matching WS and pial coordinates) for each streamline end (with K-means clustering of MCCs for speed - bottom row, 1). A streamline end is considered in grey matter if it lays within the local cortical half-thickness of its MCC (bottom row, 3). Then, two intersections with WSM (one either end) are sought (bottom row, 4) at which point the intracortical portion is truncated. The optional surface-based analysis is conducted after the filtering (top row, 5).

To improve computational efficiency, all MCC are clustered with the Kmeans algorithm using squared Euclidean distances (Arthur and Vassilvitskii (2006)); finding the centroid closest to a streamline end means the closest MCC has only to be identified within the cluster of that centroid (see Appendix C for details). 
Hemisphere-hemisphere (HH) filter. The original hemispheric membership (left or right) of all cortical vertices and thus MCC is known; the hemispheric allocation of each streamline end becomes apparent once its closest MCC is identified during GG filtering. This filter acts by only selecting streamlines whose both ends reside in the same hemisphere.

Grey-white-grey (GWG) filter. After ensuring all streamlines terminate intracortically, the last filter needs only to follow streamlines back and detect escape into white matter. Streamlines travel some distance within the cortex before this escape happens (mean per subject: 3-4 mm, median: 2-3 mm), typically resulting in a non-correspondence between the MCC associated with a streamline's end and the streamline's intersection with WSM. Due to the possibility of this occurring on a subvoxel scale, and because the exact point of intersection with WSM is of interest, filtering is performed by detecting intersections between streamline segments and WSM faces instead of applying a simple white matter mask. As considering all segments of all streamlines with all WSM faces would be extremely inefficient, intersections between bounding boxes are used in the initial step to significantly restrict the search space. The pseudocode is provided below:

for each hemisphere:

for each streamline:

define the bounding box find all surface faces

within the bounding box define a bounding

box for each identified face define a bounding

box for each individual streamline segment

register intersection between face and 
segment bounding boxes for each bounding

box intersection:

check for segment-triangle intersection take the first

intersection at either end of the streamline

Segment-triangle intersection detection is performed using signed volumes but as this is approximately three times slower compared to bounding boxes (of segment and face), the latter is done first. In addition to detecting escape into white matter, GWG filter allows to associate each streamline end with its nearest WSM vertex and truncate streamlines at that point if desired (enabled in this work).

\subsection{Framework evaluation}

Evaluation was divided into the following sections. First, the effect of surface seeding was compared against a voxel-based method to establish whether it had merit for subsequent steps. Next, the roles of scanner, acquisition and voxel size on framework performance were evaluated for parameter optimisation. Finally, a set of experiments was carried out to evaluate within- and between-subject consistency of SAF tractograms obtained using the proposed framework.

\subsubsection{Data acquisition and pre-processing}

Effects of scanner, acquisition, voxel size. Parameters of interest were evaluated using a dedicated dataset (Tax et al. (2019)) of the same 14 subjects (4 males and 10 females, age range 16-30) acquired on two different 3T scanners with different maximum gradient amplitudes (MGA): Siemens Connectom (MGA=300 mT/m) and Siemens Prisma (MGA=80 
$\mathrm{mT} / \mathrm{m}$ ). Two acquisition protocols were used: "standard" (ST) and "state-ofthe-art" (SA), with the latter having higher spatial and angular resolution achieved with multiband-acquisition and the stronger gradients to shorten TE. This, in turn, enabled a higher signal-to-noise ratio per unit time for a given b-value, allowing for utilisation of higher b-values which are more sensitive to intraaxonal water displacement (Jones et al. (2018); Setsompop et al. (2013); Genc et al. (2020)). Written informed consent was given by all subjects.

Repeatability. Repeatability data from the MICRA study (Koller et al. (2020)) were used for framework evaluation. In short, after a written informed consent, brain MR data of six healthy adults (3 males and 3 females, age range 24-30) were obtained using the Siemens Connectom $(\mathrm{MGA}=300 \mathrm{mT} / \mathrm{m})$ scanner. In comparison with the previous cohort, the MICRA protocol had a lower spatial resolution but more shells, a larger maximum b-value, and a larger total number of non-collinear directions (Table 2). Each participant was imaged five times using the same protocol within a two-week period at approximately the same time of day.

Pre-processing. Acquisition parameters for all protocols are summarised in Table 2. Spin-echo echo-planar dMRI images were corrected for slicewise intensity outliers (Sairanen et al. (2018)), signal drift (Vos et al. (2017)), Gibbs artifact (Kellner et al. (2016)), eddy current distortion and motion artifact (Andersson and Sotiropoulos (2016)), echo-planar image distortion (Andersson et al. (2003)), and gradient non-linearities (Glasser et al. (2013); 
Rudrapatna et al. (2018)). Pre-processed data were upsampled to $1 \times 1 \times 1$ mm3 (Dyrby et al. (2014)) and, for parameter evaluation, downsampled to $2 \times 2 \times 2 \mathrm{~mm} 3$. Diffusion tensor estimation in each voxel was performed with nonlinear least squares. The fODF (Tournier et al. (2007)) was derived using 3-tissue response function estimation (Dhollander et al. (2016)) and subsequent multi-shell multi-tissue constrained spherical decomposition (Jeurissen et al. (2014)) with harmonic fits up to the eighth order. The quality of the pre-processing steps as well as fODFs were visually confirmed for all subjects.

\begin{tabular}{|c|c|c|c|c|c|}
\hline Parameters & $\mathrm{ST}-300 \mathrm{mT} / \mathrm{m}$ & $\mathrm{SA}-300 \mathrm{mT} / \mathrm{m}$ & $\mathrm{ST}-80 \mathrm{mT} / \mathrm{m}$ & $\mathrm{SA}-300 \mathrm{mT} / \mathrm{m}$ & MICRA \\
\hline Scanner & Siemens Connectom & Siemens Connectom & Siemens Prisma & Siemens Prisma & Siemens Connectom \\
\hline Resolution (mm3) & $2.4 \times 2.4 \times 2.4$ & $1.2 \times 1.2 \times 1.2$ & $2.4 \times 2.4 \times 2.4$ & $1.5 \times 1.5 \times 1.5$ & $2 \times 2 \times 2$ \\
\hline Directions (per b-value) & 30 & 60 & 30 & 60 & $\#$ \\
\hline \multirow[t]{6}{*}{ b-values (s/mm2) } & 1200 & 1200 & 1200 & 1200 & 200 \\
\hline & 3000 & 3000 & 3000 & 3000 & 500 \\
\hline & & 5000 & & 5000 & 1200 \\
\hline & & & & & 2400 \\
\hline & & & & & 4000 \\
\hline & & & & & 6000 \\
\hline TE/TR (ms) & $89 / 7200$ & $68 / 5400$ & $89 / 7200$ & $80 / 4500$ & $59 / 3000$ \\
\hline
\end{tabular}

\# MICRA dataset had 20, 20, 30, 61, 61, 61 noncollinear direction per b-value, respectively.

Table 2: Acquisition parameters utilised for dMRI sequences in the two cohorts studied.

Anatomical data (Siemens MPRAGE1 sequence, voxel size: $1 \times 1 \times 1 \mathrm{~mm} 3$, TR/TE 2300/2.81 ms) were run through the FreeSurfer 7.1 package (Fischl (2012)) which includes standard T1-weighted volume pre-processing steps. For the repeatability cohort, the longitudinal stream designed for repeated acquisitions was used (Reuter et al. (2012)). One subject lacked a 
T1weighted volume for one of the sessions; instead, the within subject template (referred to as "base" in the longitudinal stream) was used, resulting in a total of six "base" and twenty-nine final (referred to as "long") sets. The quality of produced surface meshes was visually inspected at every step and corrected where necessary as per the standard FreeSurfer protocol. dMRI-derived fractional anisotropy (FA) volumes were nonlinearly registered to FreeSurfer T1-derived "brain" volumes using ANTs; coordinates of surface vertices were then brought into DWI space using the inverse transform and registration quality was visually confirmed in each case. An average subject was created for group analyses from the six "base" sets with FreeSurfer's make average subject command and used as a common space template for

all "long” sets (surface co-registration done with surfreg).

\subsubsection{Effects of surface seeding}

Choice of benchmark and tractogram generation. It was hypothesised that voxel masks result in less even seed placement hence biasing the spatial distribution of the resultant streamlines. Additionally, voxel-based filtering can lead to smaller regions being excluded or misrepresented due to the discrete nature of the voxel grid (Appendix A). To investigate how surface seeding compared to voxel-based methods, the established MRtrix ACT/GMWMI was used for benchmarking.

To approximate the tractogram generated with surface seeding, MRtrix ACT/GMWMI pipeline was modified in the following way. First, FreeSurfer's “aseg" volume was transformed to dMRI space using the already available registration and nearest neighbour interpolation (preserving segmentation 
labels). This acted as the input into MRtrix's FreeSurfer-based five-tissuetype (5TT) segmented tissue image generation algorithm (Smith et al. (2012)). The 5TT image was then manipulated such that the cerebellar cortex and the amygdala/hippocampus were excluded from the grey matter volume (matching the cortical areas used in surface seeding), while the deep nuclei as well as the ventricles were added to the white matter volume with their original volumes set to null. The manipulation effectively forced all streamlines to start and end at the neocortex. Following this, a grey matter-white matter interface (GMWMI) volume was generated and seeding performed in the usual way until the total number of streamlines for ACT/GMWMI matched the same number generated with surface seeding (see subsubsection 3.1.1); analogously, streamline length was confined to $\leq 40$

$\mathrm{mm}$.

Tractogram comparison. The initial tractograms generated with the two methods looked very different (not shown) because surface seeding on its own does not use anatomical priors as streamline termination/rejection criteria; however, after GG filtering the compartments of the white matter occupied by streamlines became comparable (Appendix D). For these experiments, only the grey-grey filter was employed as it carried out the majority of the filtering and the difference in this filter's outcome drastically affected other filters' performance.

First, coordinates of the seeds that resulted in streamlines were recorded and plotted against the white matter surface mesh to provide a qualitative assessment of seed distribution. Next, to evaluate distribution of streamlines near the cortex, a single voxel-thick mask was created using the 
outer voxels of the white matter. The mask was applied to the normalised track density imaging (TDI) maps (Calamante et al. (2010)) produced using the two methods and only the voxels that were present in both were further considered. TDI maps were visually inspected, after which variation in the distribution of the subcortical streamlines' density was further assessed by plotting histograms of voxel TDI values normalised to 0-1 range. This provided an overview of how variable subcortical streamline density was on a wholebrain level. Local variability was then assessed by recording the coefficient of variation of normalised TDI values within each voxel's neighbourhood (6-connected voxel patches up to $5 \times 5 \times 5$ in size) and similarly plotting these as histograms. Finally, GG filter was applied to the ACT/GMWMI-generated tractograms and the difference was evaluated on the following characteristics: (1) number of streamlines surviving filtering; (2) cortical coverage (proportion of vertices with streamlines); (3) number of streamlines associated with each vertex; (4) proportion of "covered" surface representing gyri (estimated as proportion of streamline-linked surface vertices with a negative FreeSurfer "sulc" value).

\subsubsection{Effects of scanner, acquisition, voxel size}

For this set of experiments, the end point of seeding was generation of $5 \mathrm{M}$ streamlines (as opposed to 5M seeds) before filtering could begin. This allowed for a better appreciation of the interaction between the parameters in question and the filtering.

Fibre orientation distribution functions. Scanner and sequence effects on fODF configuration were evaluated visually. For this comparison only, all 
four datasets of a subject were registered to the same (subject-specific) T1weighted volume.

Streamline angle at cortex. One of the manifestations of gyral bias is drastically different angulation of streamline origins/terminations with respect to the cortical surface depending on their position along the gyral blades. A typical distribution will see streamlines near-radial to the surface in the gyral crowns and near-tangential along the banks and the sulcal fundi (Cottaar et al. (2021); Van Essen et al. (2014)). To investigate how the differences in fODFs between scanners and sequences might affect this pattern, the angle between the first streamline segment and the cortex was plotted against the position along the gyral blade.

Anatomical distribution of connections. To determine which type of SAF streamlines were most affected by the parameters of interest, position along the gyral blades was split into five zones (from sulcal fundi to gyral crowns) of equal surface areas, and undirected connectivity matrices were plotted with edge weights determined by streamline counts similar to the recent work by Cottaar et al. (2021).

Tractogram comparison. After inspecting tractograms visually (all registered affinely to Connectom/SA/1 $\mathrm{mm}^{3}$ space), the effect of scanner, acquisition and voxel size was additionally investigated using linear mixed modelling with all three modelled as fixed effects and participants as the random effect. The outcomes were surface area seeded (\%), surface area with SAF streamlines (\%), mean number of streamlines per vertex, the 
overall number of SAF streamlines surviving the filtering and the mean streamline length.

\subsubsection{Tractogram characteristics and assessment of consistency}

The following complementary approaches were chosen to evaluate the generated SAF tractograms: (1) general streamline metrics; (2) volumebased track density imaging (TDI) maps; (3) surface-based projections of streamline metrics. The focus of these assessments was to provide a description of the tractograms and to test their consistency within and between subjects rather than producing inferences about the physiological properties of SAF themselves.

General streamline measures. SAF tractograms were assessed and compared using the following criteria: (1) streamline count; (2) mean streamline length; (3) mean streamline FA derived by sampling respective FA volumes along each streamline with MRtrix's tcksample command (mean FA across each streamline).

TDI maps. TDI maps for each session were generated with MRtrix's tckmap command. The maps were compared in average subject space by applying the previously obtained dMRI-to-T1 transform followed by a "base-to-average" transform (concatenated and performed in a single step using ANTs).

Surface-based analysis. Each streamline terminated at two WSM vertices (one per end) allowing streamline-related metrics to be recorded at these vertices in native space. This enabled the use of surface-based registration for comparisons, which due to the complexities of cortical folding, can be superior to volume-based registration for these superficial structures. The 
following metrics were thus recorded: (1) number of streamlines per vertex; (2) cortical coverage (binarised version of the former); (3) mean streamline length per vertex; (4) mean FA per streamline per vertex. The latter can be thought of as being conceptually similar to uniform sampling of FA (or, indeed, any scalar) along the surface, with the exception that the size and shape of the sampling kernel changed informed by the streamlines at the vertex being sampled, and the sampling occurred exceptionally in the white matter.

Results were saved in FreeSurfer "curv" format (Appendix G). Two types of analysis were performed: (1) using average values for all vertices of both hemispheres; (2) directly comparing surfaces on a per-vertex basis per hemisphere. For the latter, corresponding "curv" files were stacked (mris_preproc) and smoothed (mri_surf2surf) at $5 \mathrm{~mm}$ full-width at halfmaximum (FWHM). Smoothing is commonly used in neuroimaging to boost signal-to-noise ratio, alleviate registration misalignment, and improve normality of residuals (Jones et al. (2005)). As per-vertex testing was not sensitive to between-vertex interactions, smoothing provided an alternate means to account for these interactions. For all surface-based analyses, only the cortical surface (excluding the "medial wall" label) was studied.

\subsubsection{Statistical analysis}

To compare surface seeding with ACT, a two-tailed paired sample t-test was used. In subsequent experiments, consistency of SAF tractograms was evaluated by examining metric reproducibility, between-subject variability and reliability. The former two were calculated using within $\left(\mathrm{CV}_{\mathrm{W}}\right)$ and between $\left(C_{B}\right)$ subject coefficients of variation, respectively (Laguna et al. 
(2020)). Reliability of metrics was characterised using single measurement intraclass correlation coefficient for absolute agreement ICC $(3,1)$ with subject effects modelled as random and session effects fixed (McGraw and Wong (1996)). The data were formulated with a linear mixed-effects model (Chen et al. (2018)). For voxel-based (TDI) and surface-based analysis, $\mathrm{CV}_{\mathrm{W}}$, $\mathrm{CV}_{\mathrm{B}}$ and ICC were calculated at each voxel/vertex. All statistical analyses were performed in MATLAB $2015 \mathrm{a}$.

\subsection{Data/code availability statement}

- Data: please refer to Koller et al. (2020) for access to the test-retest data, and to Tax et al. (2019) for access to the cross-scanner and cross-protocol diffusion MRI data harmonisation database.

- Code: the MATLAB code for filtering of SAF and interfacing with the surface will be made available upon publication at: https://github.com/dmitrishastin/SAF

\section{Results}

\subsection{Effects of surface seeding}

Seed distribution. The distribution of seeds that resulted in streamlines is illustrated in relation to the WSM in the top row of Figure 2. This demonstrates that surface seeding produced a smaller number of unique seeds (being confined to the WSM vertices only) yet appeared to provide a more consistent GMWMI coverage observed in both gyri and sulci.

Subcortical streamline density. Inspecting the voxel-thick subcortical TDI maps, surface-based method appeared to have a smaller variation in the 
number of streamlines although there were regions that were better "filled" using the voxel-based approach too (Figure 2B, see Appendix E for 3D views). Histogram plotting (Figure 2, top graphs) showed that while voxelbased approach performed very consistently within and between subjects, it

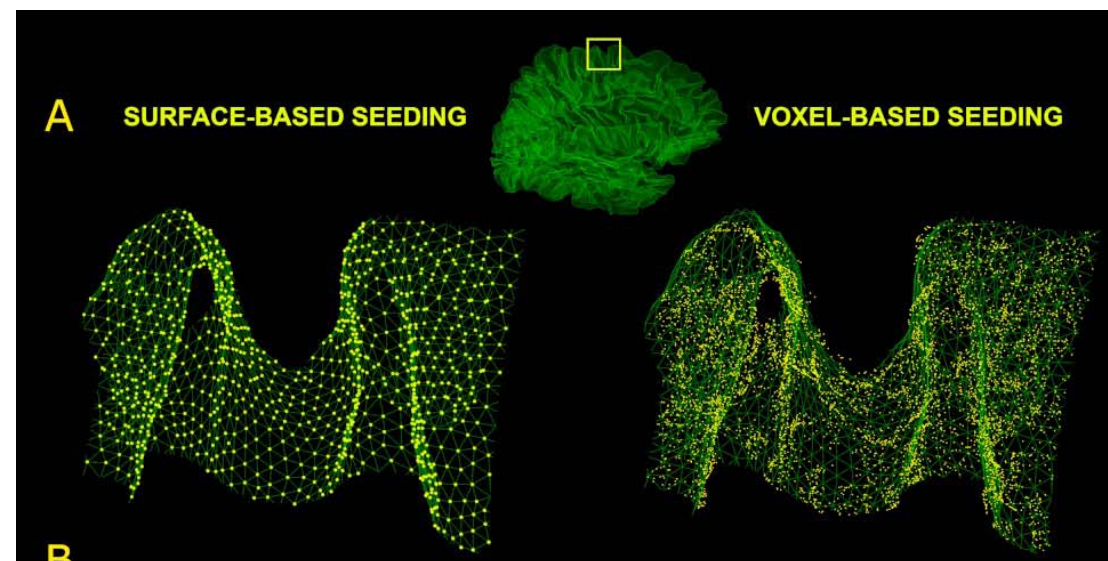

B

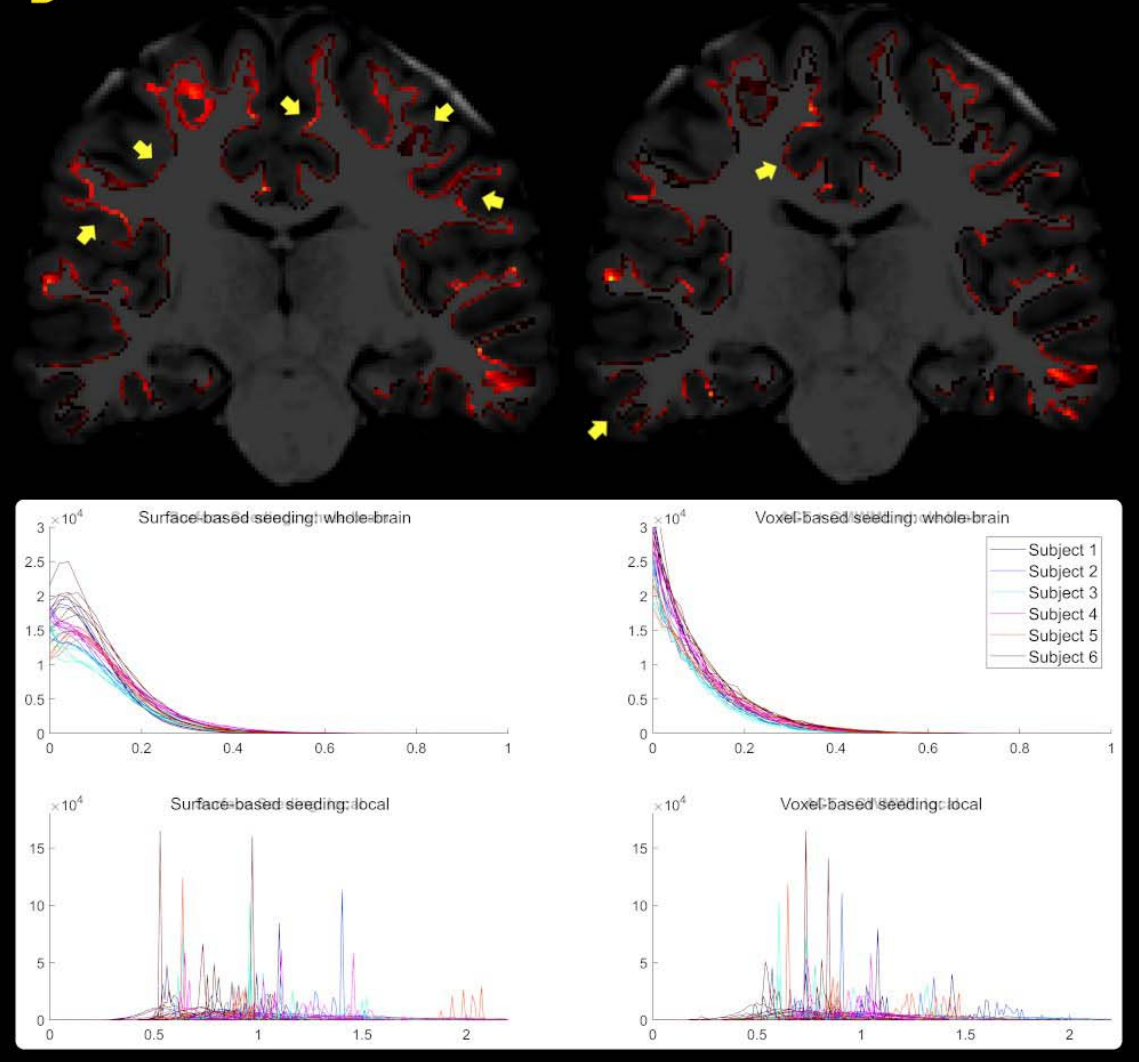


Figure 2: Comparison of surface- and voxel-based seeding strategies and their effects on subcortical streamline density. A: distribution of seeding coordinates with each method (yellow) related to a section of the WSM (green) in the region of the paramedian premotor cortex (surface mesh with the region-defining box in the middle). Surface seeding appears to achieve a more spatially uniform distribution and places seeds directly on the mesh (by definition). B: Normalised maps of subcortical streamline densities obtained with the two methods. Yellow arrows point to some areas that differ between the methods. Top graph: histograms of normalised subcortical voxel TDI values with both methods. Bottom graph: histograms of coefficients of variance for each subcortical voxel of TDI values computed over that voxel's neighbourhood. Each subject's histograms were coloured consistently.

resulted in a very large number of voxels that had low streamline counts (mean normalised value: 0.101). Surface-based method, on the other hand, was less consistent but resulted in more densely populated voxels (mean normalised value: 0.1170) $(\mathrm{p}<0.001)$. Comparison of local densities produced distributions that were very inconsistent within or between subjects with either method (Figure 2, bottom graphs). Mean coefficient of variance (calculated for each tractogram) did not differ significantly with either method (surface-based: 0.9984, voxel-based: 0.9320, p=0.415) suggesting that method choice did not change the local variation in the number of streamlines subcortically.

GG-filtered tractograms. Compared to the voxel-based method (Table 3), surface seeding resulted in a slightly larger number of streamlines surviving GG filtering $(\mathrm{p}=0.006)$. Importantly, 33\% more MCCs were covered with streamlines $(\mathrm{p}<0.001)$ and each MCC was associated with a 2.4 -fold increase in streamlines on average $(\mathrm{p}<0.001)$. This difference was likely influenced by the more consistent seed placement with surface seeding and the fact that with the voxel-based method, streamlines did not 
propagate into grey matter and occasionally even left a superficial rim of unfilled white matter (Appendix D), likely due to the incorporated anatomical priors. The latter would have prevented subsequent GG filtering from accepting some streamlines. Decreasing the fODF amplitude threshold during tracking did not improve this result significantly. In contrast, surface seeding allowed tracking to propagate in both directions with non-white matter sections truncated later at the GWG filtering step (not performed in this experiment). However, the expected gyral bias was noticed with surface seeding as streamlines associated with more MCCs in gyri than in sulci whereas the reverse was true for the voxel-based method $(p<0.001)$. As subsequent experiments suggest (Figure 4, Figure 5), higher angular and spatial resolution of data translate to more streamlines being retained after filtering in gyral regions corresponding to more prominent fODFs in these areas (Figure 3). Whether this effect is desired remains to be investigated.

\begin{tabular}{|c|c|c|c|c|c|}
\hline & \multicolumn{2}{|c|}{ Surface Seeding } & \multicolumn{2}{|c|}{ Voxel Seeding ${ }^{3}$} & \multirow[b]{2}{*}{ p4 } \\
\hline & Mean & SD & Mean & SD & \\
\hline Number of streamlines generated & $4.5 \mathrm{M}$ & $0.1 \mathrm{M}$ & $4.5 \mathrm{M}$ & $0.1 \mathrm{M}$ & matched \\
\hline Number of streamlines after GG filter ${ }^{1}$ & $1.1 \mathrm{M}$ & $52 \mathrm{~K}$ & $1.0 \mathrm{M}$ & $64 \mathrm{~K}$ & 0.006 \\
\hline Cortical coverage $(\%)^{2}$ & 88.36 & 2.14 & 55.72 & 1.08 & 0.000 \\
\hline Termination density (streamlines/vertex) ${ }^{2}$ & 18.39 & 2.55 & 7.68 & 1.06 & 0.000 \\
\hline Prevalence of gyri in the covered surface $(\%)^{2}$ & 52.77 & 0.65 & 47.95 & 0.58 & 0.000 \\
\hline \multicolumn{6}{|l|}{${ }^{1}$ Streamlines starting and ending in the neocortex } \\
\hline \multicolumn{6}{|c|}{2 Calculated at MCC, averaged over all vertices of both hemispheres } \\
\hline${ }^{3}$ Using FreeSurfer algorithm, modified only to & he cortic & bbon & & & \\
\hline
\end{tabular}


Table 3: Results of filtering after tracking from the surface vertices versus tracking using a voxel-based method for the same initial number of streamlines. Seeding from the surface leads to more efficient subsequent filtering and a larger cortical coverage.

\subsection{Effects of scanner, acquisition, voxel size}

Fibre orientation distribution functions. While the shape and direction of the glyphs did not appear to differ significantly at the level of the corona radiata, it was noted that at the subcortical grey-white matter interface and further into the cortex the glyphs from the Connectom scanner were more consistently perpendicular to the cortical surface (particularly so with the SA data, see Figure 3). This was likely the result of higher angular and/or spatial resolution (Vos et al. (2016)).

Streamline angle at cortex. The shape of the distribution appeared similar irrespective of the parameters, with least acute angles seen near the gyral crowns, a near-parallel course along the banks then a slight increase in angles again near the sulci. For all datasets, the crowns and the fundi saw a greater dispersion of angles compared to the banks. On the other hand, the three parameters affected the overall number of streamlines, with Connectom scanner, SA and a smaller voxel size all resulting in a larger proportion of streamlines terminating closer to the gyral crowns and increasing the average angle (Figure 4).

Anatomical distribution of connections. Connectivity matrices confirmed that the increase in streamline counts associated with Connectom scanner, SA acquisition and a smaller voxel size resulted in an overall increase in streamlines. However, this increase was not homogenous with more 
connections observed between gyral crowns and the gyral walls next to them (Figure 5). While the reason behind the preference for gyri in this case was not directly investigated, all datasets started with the same number of streamlines meaning that in this group, fewer streamlines were filtered out.

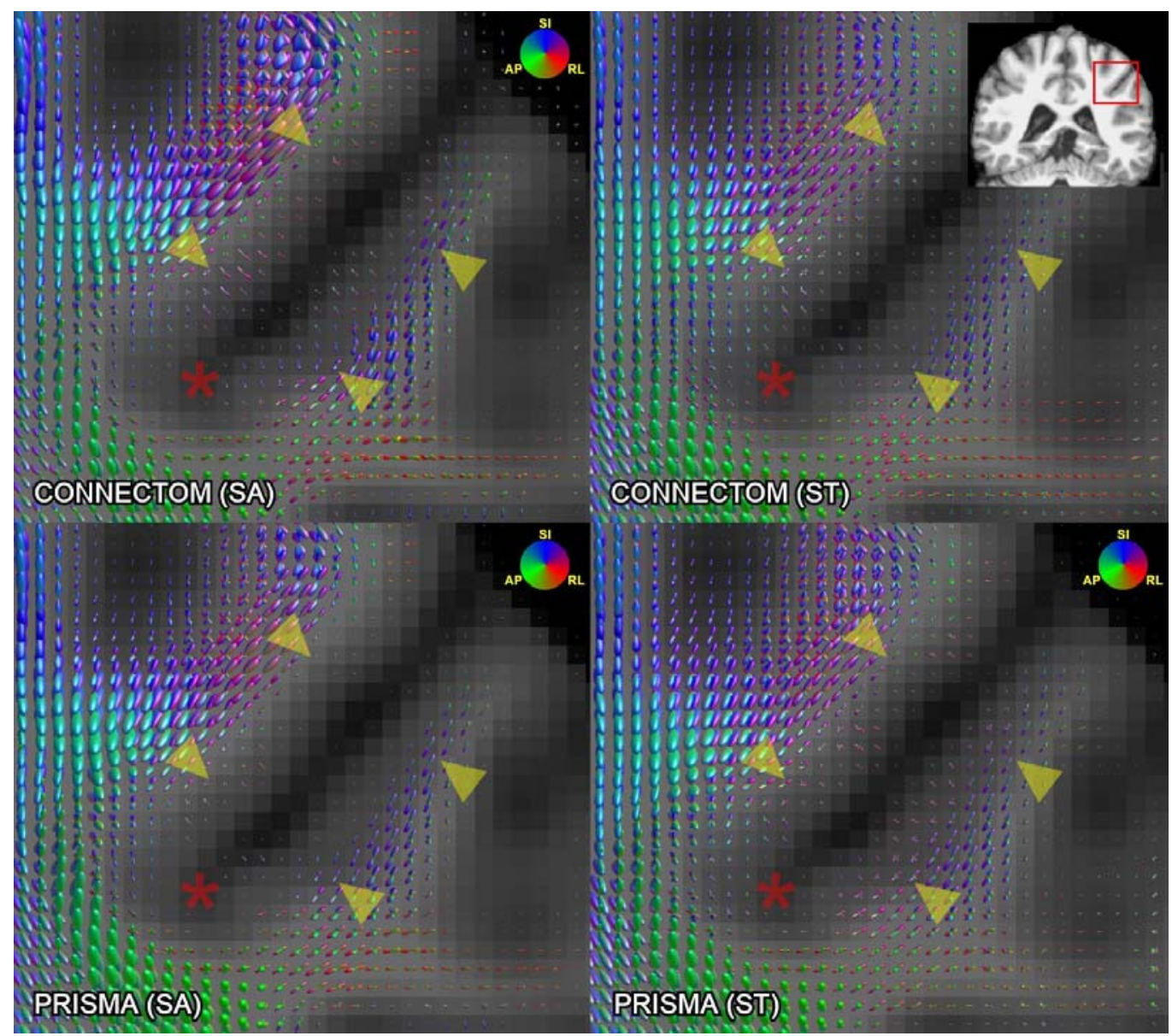

Figure 3: Comparison of fODFs ( $\max =8$, scale=2) generated using Connectom (top row) and Prisma (bottom row), "state-of-the-art" (left column) and "standard" (right column) acquisitions for the same subject on a $1 \mathrm{~mm}^{3}$ isotropic voxel grid, affinely co-registered to the same T1-weighted volume. SI, superior-inferior. AP, anterior-posterior. RL, right-left. 

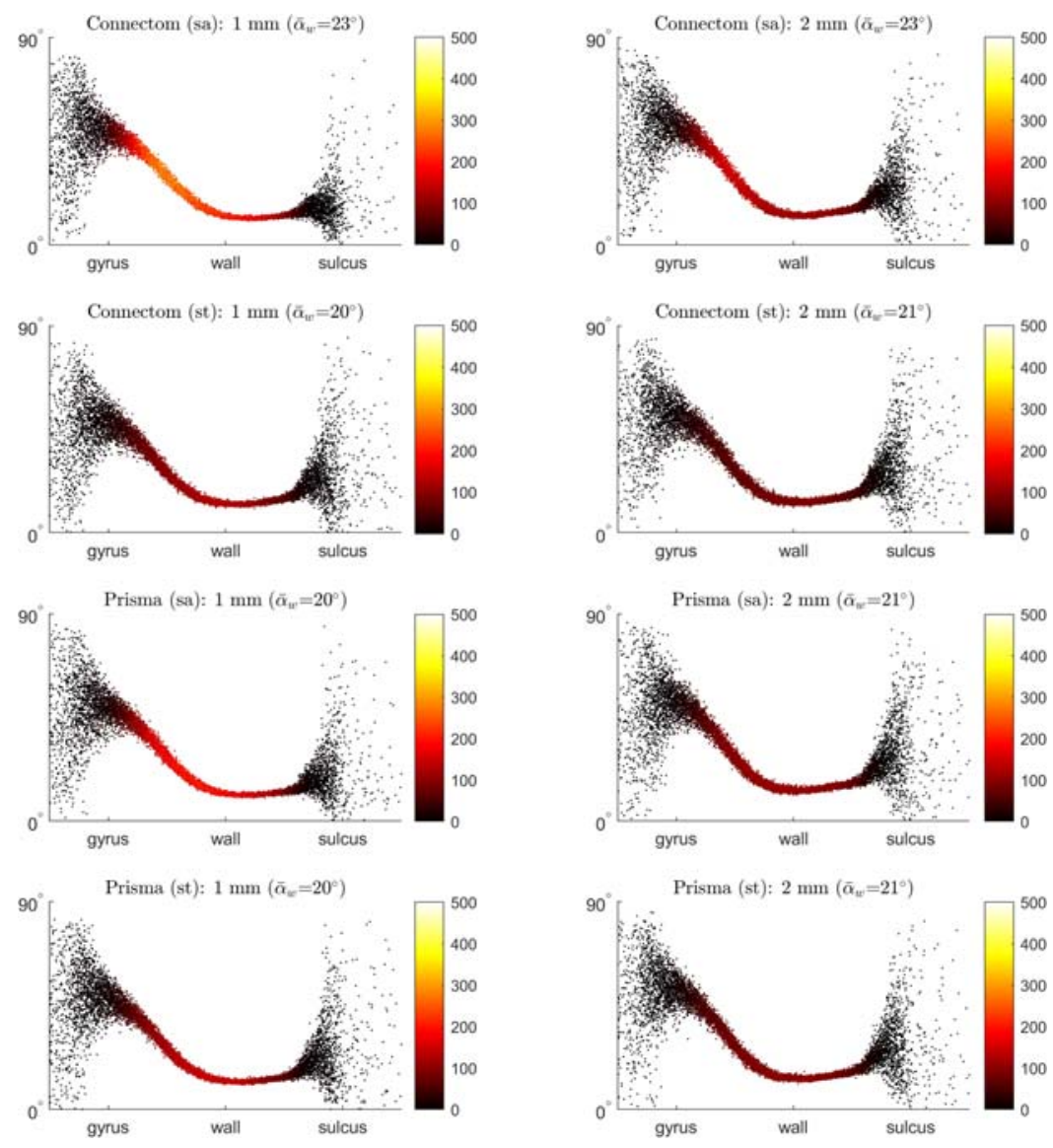

Figure 4: Effect of scanner, acquisition, and voxel size on the angle between the terminating segment of a streamline and the overlying cortical mesh, depending on the former's location along the gyral blade. Connectom scanner, "state-of-the-art" sequence and smaller voxel size all seemed to increase the number of streamlines largely terminating between walls and crowns of the gyri.

It is possible that due to a more diverse distribution of fODFs near the greywhite interface (Figure 3), more streamlines reached the cortex and survived the filtering. Figure 3 also reveals a larger magnitude of the glyphs closer to gyral crowns relative to those in the corona radiata with SA sequences (top and left sides of each quadrant, respectively), further hinting 
at why more streamlines were observed in this region. It is likely that with the Prisma scanner, ST acquisition and $2 \mathrm{~mm}^{3}$ voxels, fewer streamlines terminated in the gyral cortex leading to them being excluded at the GG filter stage.

Tractogram comparison. There was a very clear difference in tractogram appearance depending on the choice of scanner, acquisition, and voxel size (Figure 6). Larger voxel size generally translated to a much poorer coverage, with long thin gyral blades and sulcal fundi particularly affected. Similarly, the "standard" sequence appeared to cover long thin gyral blades considerably less. The choice of scanner appeared to have a more subtle but clearly observed influence, with Connectom tractograms appearing denser and covering subcortical white matter more uniformly. With variable extent, all datasets had gyri with few-to-none streamlines in the central part of the crown as streamlines tended to preferentially adhere to walls.

The linear mixed model revealed that voxel size had by far the strongest impact on all outcomes, with smaller voxels resulting in larger area covered by seeds and streamlines alike, and with higher overall streamline numbers (Table 4). Larger voxel size caused streamlines to be a few $\mathrm{mm}$ longer on average. To assess whether these differences were linked to step size during tracking, a small number of datasets were re-processed with $2 \mathrm{~mm}^{3}$ voxels and half the step size. This, however, did not result in significant differences for any of the outcomes (data not shown). 

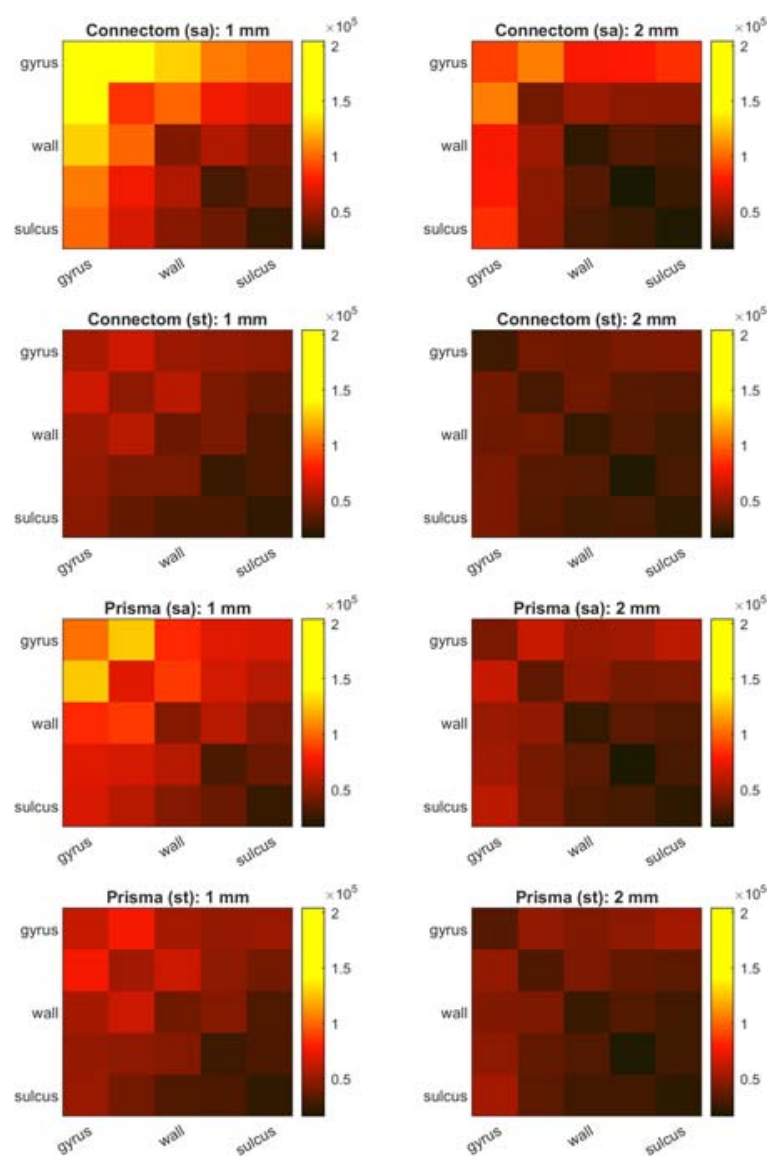

Figure 5: Effect of scanner, acquisition, and voxel size on SAF connectivity between different positions along gyral blades. Cortical surface was divided into five zones with equal areas from sulcal fundi to gyral crowns. Connectivity matrices suggest that Connectom, "state-of-the-art" sequence, small voxel size increased the overall number of connections but particularly so between gyral crowns and the surrounding areas.

Scanner type only influenced the number of streamlines, mean streamlines per vertex and mean streamline length, and for these parameters its effect was noticeably smaller compared to that of acquisition or voxel size. Acquisition influenced all four outcomes. Paradoxically, the ST sequence seemingly increased, not decreased the surface area seeded (this was also 


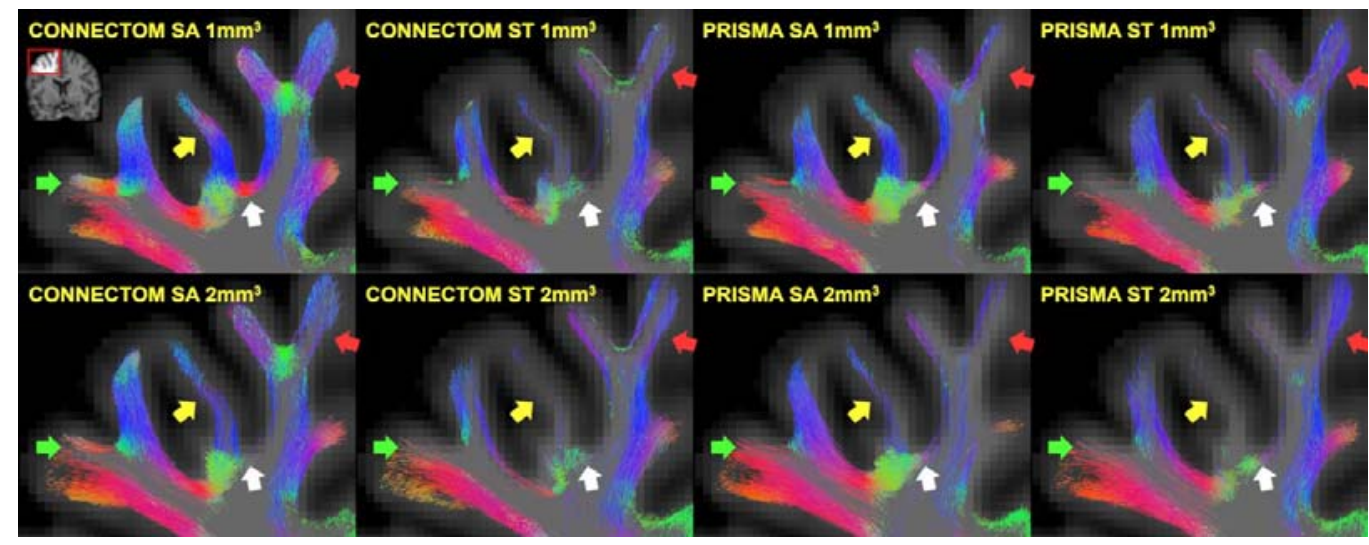

Figure 6: Appearances of SAF tractograms depending on the choice of scanner, acquisition, and voxel size. A region from the right frontal lobe is shown in coronal plane (inset in the right upper corner). Tractogram slices are $1 \mathrm{~mm}$ thick. The differences between the datasets in the distribution of streamlines in sulci (white arrow), gyri (red arrow) and, in particular, long thin gyri (yellow arrow) and the central part of the wider gyral crowns (green arrow) are obvious.

observed visually particularly with the Prisma datasets). The cause for the latter requires further investigation, but the direction of this effect did not translate to other outcomes.

\begin{tabular}{lcccc}
\hline Surface Area Seeded (\%) & Beta & Lower 95\% & Upper 95\% & p-value \\
\hline Intercept & 82.96 & 80.75 & 85.18 & $<0.001$ \\
Scanner & 0.02 & -1.13 & 1.16 & 0.979 \\
Acquisition type & 3.85 & 2.71 & 4.99 & $<0.001$ \\
Voxel size & -10.4 & -11.55 & -9.26 & $<0.001$ \\
\hline Surface area with SAF streamlines (\%) & & & \\
\hline Intercept & 67.86 & 64.93 & 70.79 & $<0.001$ \\
Scanner & 0.16 & -1.55 & 1.87 & 0.852 \\
Acquisition type & -3.74 & -5.45 & -2.03 & $<0.001$ \\
Voxel size & -11.65 & -13.36 & -9.94 & $<0.001$ \\
\hline
\end{tabular}




\begin{tabular}{lcccc}
\hline \multicolumn{1}{l}{ Streamlines/vertex (mean) } & & & & \\
\hline Intercept & 8.68 & 8.25 & 9.12 & $<0.001$ \\
Scanner & -0.77 & -1.13 & -0.40 & $<0.001$ \\
Acquisition type & -2.49 & -2.86 & -2.12 & $<0.001$ \\
Voxel size & -2.43 & -2.80 & -2.07 & $<0.001$ \\
\hline Number of SAF streamlines & & & & \\
\hline Intercept & $1.11 \mathrm{M}$ & $1.06 \mathrm{M}$ & $1.17 \mathrm{M}$ & $<0.001$ \\
Scanner & $-97 \mathrm{~K}$ & $-145 \mathrm{~K}$ & $-50 \mathrm{~K}$ & $<0.001$ \\
Acquisition type & $-321 \mathrm{~K}$ & $-369 \mathrm{~K}$ & $-274 \mathrm{~K}$ & $<0.001$ \\
Voxel size & $-312 \mathrm{~K}$ & $-360 \mathrm{~K}$ & $-265 \mathrm{~K}$ & $<0.001$ \\
\hline Mean streamline length & & & & $<0.001$ \\
\hline Intercept & 16.89 & 16.59 & 17.22 & $<0.001$ \\
Scanner & 0.29 & 0.14 & 0.43 & $<0.001$ \\
Acquisition type & -1.09 & -1.24 & -0.95 & $<0.01$ \\
Voxel size & 3.31 & 3.16 & 3.46 & $<$ \\
\hline
\end{tabular}

Table 4: Effect of scanner, acquisition and voxel size on the final SAF tractogram estimated using linear mixed modelling. Connectom scanner, SA acquisition, $1 \mathrm{~mm}^{3}$ voxels were used as reference.

\subsection{Assessment of SAF tractograms}

Framework runtime was 4 hours on average with parallel processing (12 CPUs) enabled. Tractograms generated using the repeatability cohort appeared anatomically consistent and no manual pruning was required (Figure 7). On average, 20\% of the original streamlines survived the filtering. 


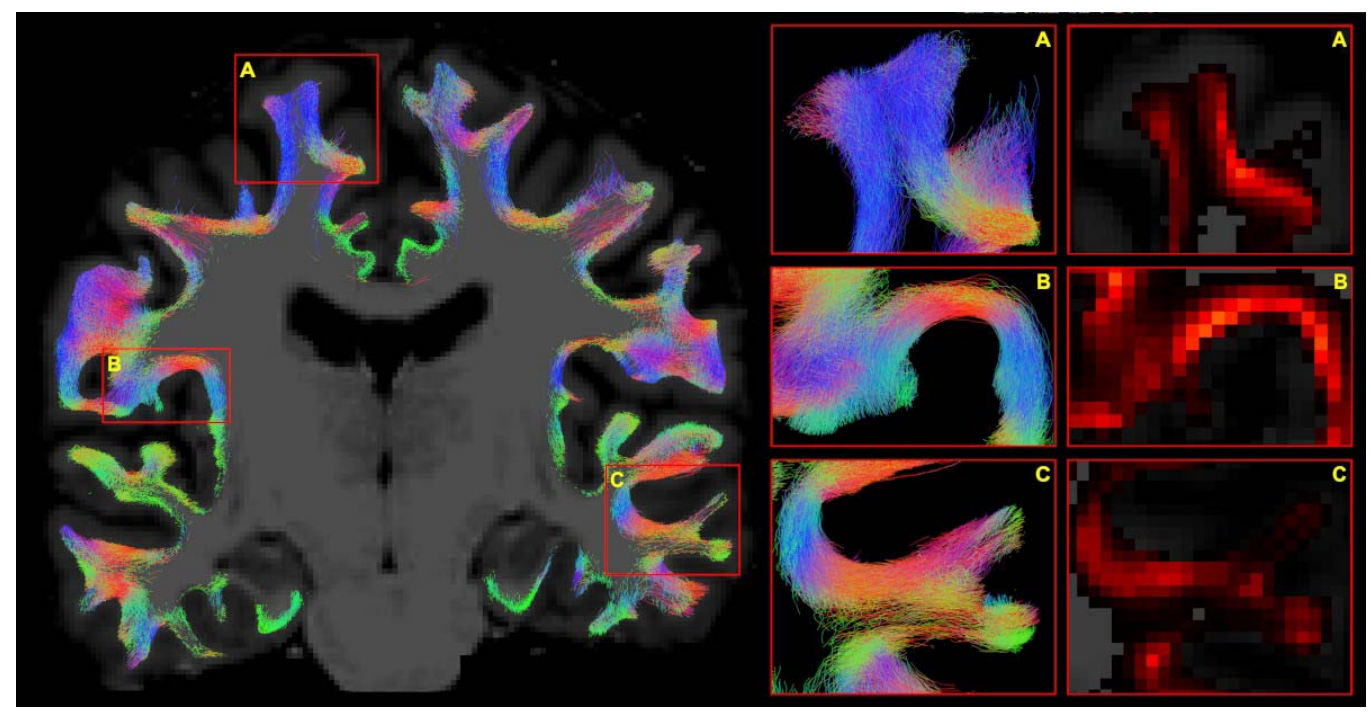

Figure 7: Final appearance of SAF tractogram following the filtering process. Left: SAF streamlines overlaid on the T1-weighted volume in dMRI space (coronal view, $1 \mathrm{~mm}$ thick slice). Right, first column: SAF bundles will often deviate from the orthogonal planes in their course. $5 \mathrm{~mm}$ thick slices are provided for a better appreciation of their extent. Right, second column: TDI maps of the same regions are provided. Regions on the left are represented in the right-hand columns under matching letters.

For the most part, SAF appeared to course in a bundled fashion and there appeared to be numerous mixing of bundles as highlighted by directionencoded colourmaps (Figure 7A and B, first column). Mean streamline length was $19.11 \pm 0.16 \mathrm{~mm}$ after trimming the intracortical portions. The average FA per streamline was $0.31 \pm 0.01$. Tractograms covered $87.27 \pm 1.78 \%$ of WSM vertices with $6.94 \pm 1.10$ streamlines per vertex. Mean streamline length and mean FA per vertex were similar to overall mean length and FA, respectively. The range of values on the surface in a single subject can be seen in Appendix G. Coverage of both gyral and sulcal surfaces appeared satisfactory (Figure 7, second column, also Appendix F). 
The overall proportion of streamlines terminating in gyri was $59.21 \pm 1.30 \%$, supporting the presence of gyral bias.

Compared to the Connectom/SA/1 $\mathrm{mm}^{3}$ voxel dataset from the previous experiments, the streamlines appeared to form less acute angles to the surface and there was more "mixing" of directions. The distribution of angles when plotted against position along the gyral blades appeared consistent except for less acute angles seen near the sulcal regions, and higher mean angle (Appendix F). This cohort had similar surface area seeded but had a larger area covered by streamlines post filtering. Having a comparable mean streamline length together with a lower number of streamlines per vertex and the overall number of streamlines of the same order, the tractograms in the repeatability cohort appeared more evenly distributed over the surface.

Apart from streamline length, all metric means exhibited high reproducibility $\left(\mathrm{CV}_{\mathrm{W}}\right.$ 0.51-3.56\%), low between-subject variation $\left(\mathrm{CV}_{\mathrm{B}}\right.$ 0.809.37\%), and moderate-to-high reliability (ICC 0.654-0.978). Streamline length had a very low standard deviation across the board resulting in similarly low $\mathrm{CV}_{\mathrm{W}}$ and $\mathrm{CV}_{\mathrm{B}}$ therefore producing a low ICC.

\subsection{Track density imaging maps}

Although TDI maps suggested an overall moderate-to-high reliability of the spatial distribution of streamlines (median ICC: 0.771 ), reproducibility was low (median $\mathrm{CV}_{\mathrm{W}}$ : 66.16\%) suggesting a lot of variation within subjects (Figure 8). Median $\mathrm{CV}_{\mathrm{B}}$ was $274.76 \%$ (thresholded in the figure), attesting to a very high variation between subjects when comparing TDI maps. The 
immediate subcortical areas showed the least consistency $\left(30-50 \% \mathrm{CV}_{\mathrm{W}}\right.$ with

\begin{tabular}{lccccc}
\hline Seeding and Filtering Descriptors & Mean $^{1}$ & SD $^{1}$ & $\mathbf{C V}_{\mathbf{W}}(\%)$ & $\mathbf{C V}_{\mathbf{B}}(\%)$ & ICC \\
\hline Number of streamlines generated & $4.5 \mathrm{M}$ & $39.1 \mathrm{~K}$ & 1.11 & 5.50 & 0.877 \\
Total number of streamlines retained after filtering & $0.9 \mathrm{M}$ & $20.7 \mathrm{~K}$ & 3.56 & 9.37 & 0.654 \\
\hline
\end{tabular}

Filtered Tractogram Descriptors ${ }^{2}$

\begin{tabular}{|c|c|c|c|c|c|}
\hline Streamline length (mm) & 19.11 & 0.14 & 0.99 & 0.80 & 0.000 \\
\hline Mean streamline FA & 0.31 & 0.00 & 1.28 & 5.55 & 0.845 \\
\hline \multicolumn{6}{|l|}{ Surface Data Before Filtering ${ }^{3}$} \\
\hline Cortical coverage (\%) & 88.43 & 0.70 & 1.11 & 5.42 & 0.874 \\
\hline Termination density (streamlines/vertex) & 18.38 & 0.27 & 2.07 & 3.10 & 0.985 \\
\hline \multicolumn{6}{|l|}{ Surface Data After Filtering ${ }^{4}$} \\
\hline Cortical coverage (\%) & 87.26 & 0.87 & 1.38 & 4.27 & 0.729 \\
\hline Termination density (streamlines/vertex) & 6.93 & 0.15 & 3.08 & 3.76 & 0.978 \\
\hline Mean streamline length per vertex (mm) & 17.05 & 0.25 & 1.91 & 6.20 & 0.749 \\
\hline Mean streamline FA per vertex & 0.28 & 0.01 & 2.44 & 9.03 & 0.796 \\
\hline Prevalence of gyri in the covered surface (\%) & 59.21 & 1.30 & 0.51 & 2.84 & 0.904 \\
\hline \multicolumn{6}{|l|}{1 Averaged within subjects } \\
\hline \multicolumn{6}{|c|}{2 Averaged over all SAF streamlines of both hemispheres } \\
\hline \multicolumn{6}{|c|}{${ }^{3}$ Calculated at MCC, averaged over all vertices of both hemispheres } \\
\hline
\end{tabular}

Table 5: Whole-brain metrics. Surface metrics were calculated before smoothing. Measures of consistency were calculated using mean values per session. $\mathrm{SD}$, standard deviation. $\mathrm{CV}_{\mathrm{W}}$ coefficient of variation within subjects. $\mathrm{CV}_{\mathrm{B}}$, coefficient of variation between subjects. ICC, intraclass correlation coefficient. FA, fractional anisotropy.

the outer voxels reaching $200-600 \%$, and $300-400 \% \mathrm{CV}_{\mathrm{B}}$ ), possibly as a result of cortical folding differences (manifesting in registration 
imperfections) coupled with partial volume effects or less reliable tracking on the grey-white

interface.

\subsection{Surface-based analysis}

In order to minimise the registration-related distortions and issues with cortical folding differences, streamline data were projected on the surface in

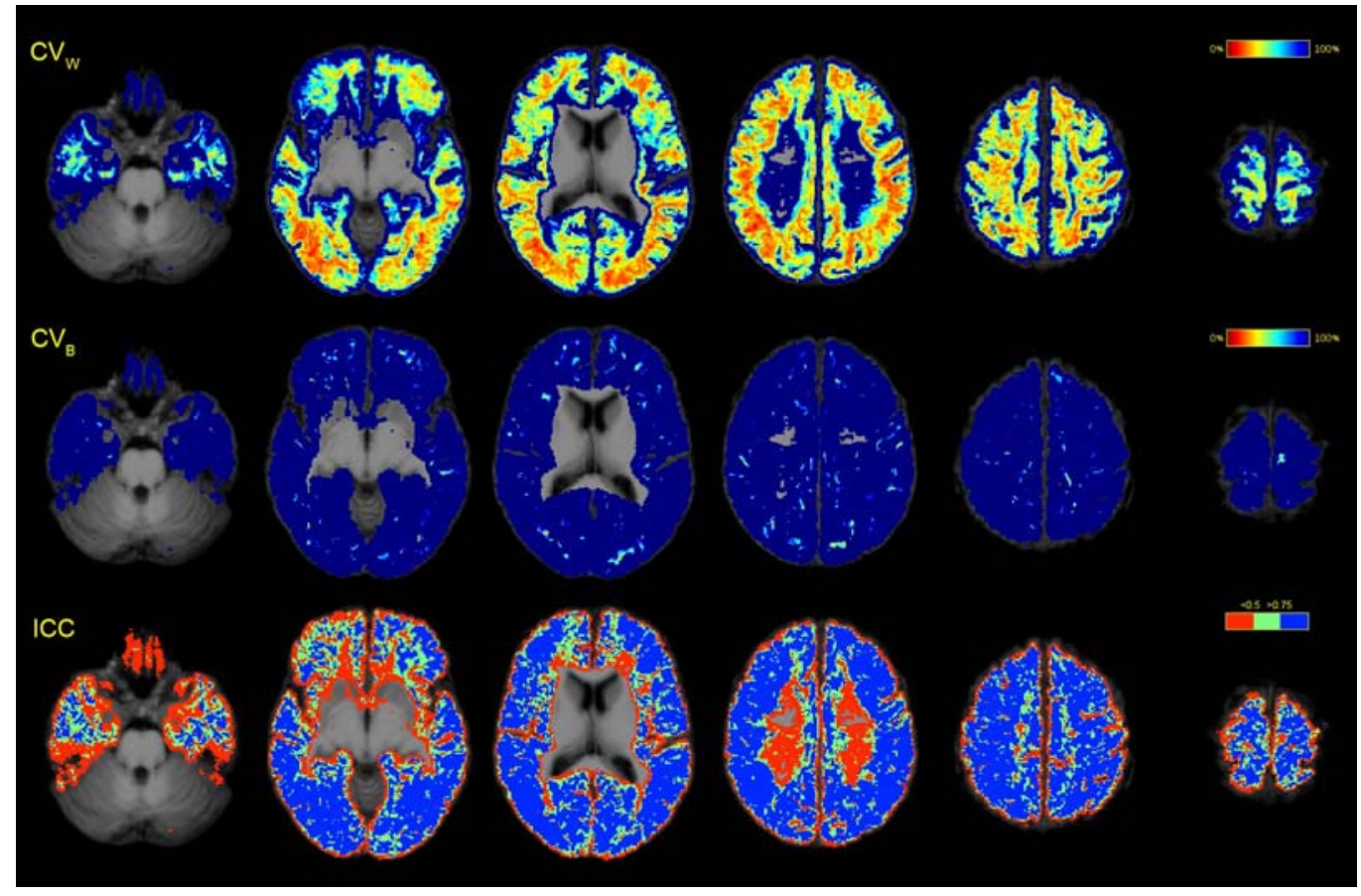

Figure 8: Repeatability of SAF using TDI map comparison in common space. All maps were superimposed on the average T1-weighted volume. Streamlines were truncated at the white matter surface before map generation. $\mathrm{CV}_{\mathrm{W}}$ and $\mathrm{CV}_{\mathrm{B}}$ were thresholded at $100 \%$. ICC was thresholded at $<0.5$ and $>0.75 . \mathrm{CV}_{\mathrm{W}}$, coefficient of variation within subjects. $\mathrm{CV}_{\mathrm{B}}$, coefficient of variation between subjects. ICC, intraclass correlation coefficient. 
native space before applying surface registration (Figure 9, also Appendix H). Analysis of termination density demonstrated moderate reliability (median ICC: 0.780) and low reproducibility (median $\mathrm{CV}_{\mathrm{W}}:$ 27.13\%) together with high between-subject variability (median $\mathrm{CV}_{\mathrm{B}}: 100.35 \%$ ), although this compared favourably to the results seen with TDI analysis. Similar ICC coefficients and improved coefficients of variation were shown for mean length per vertex (median ICC: 0.666, median $\mathrm{CV}_{\mathrm{W}}$ : 12.51\%, median $\mathrm{CV}_{\mathrm{B}}: 38.48 \%$ ) and mean FA per vertex (median ICC: 0.685, median $\mathrm{CV}_{\mathrm{W}}: 8.02 \%$, median

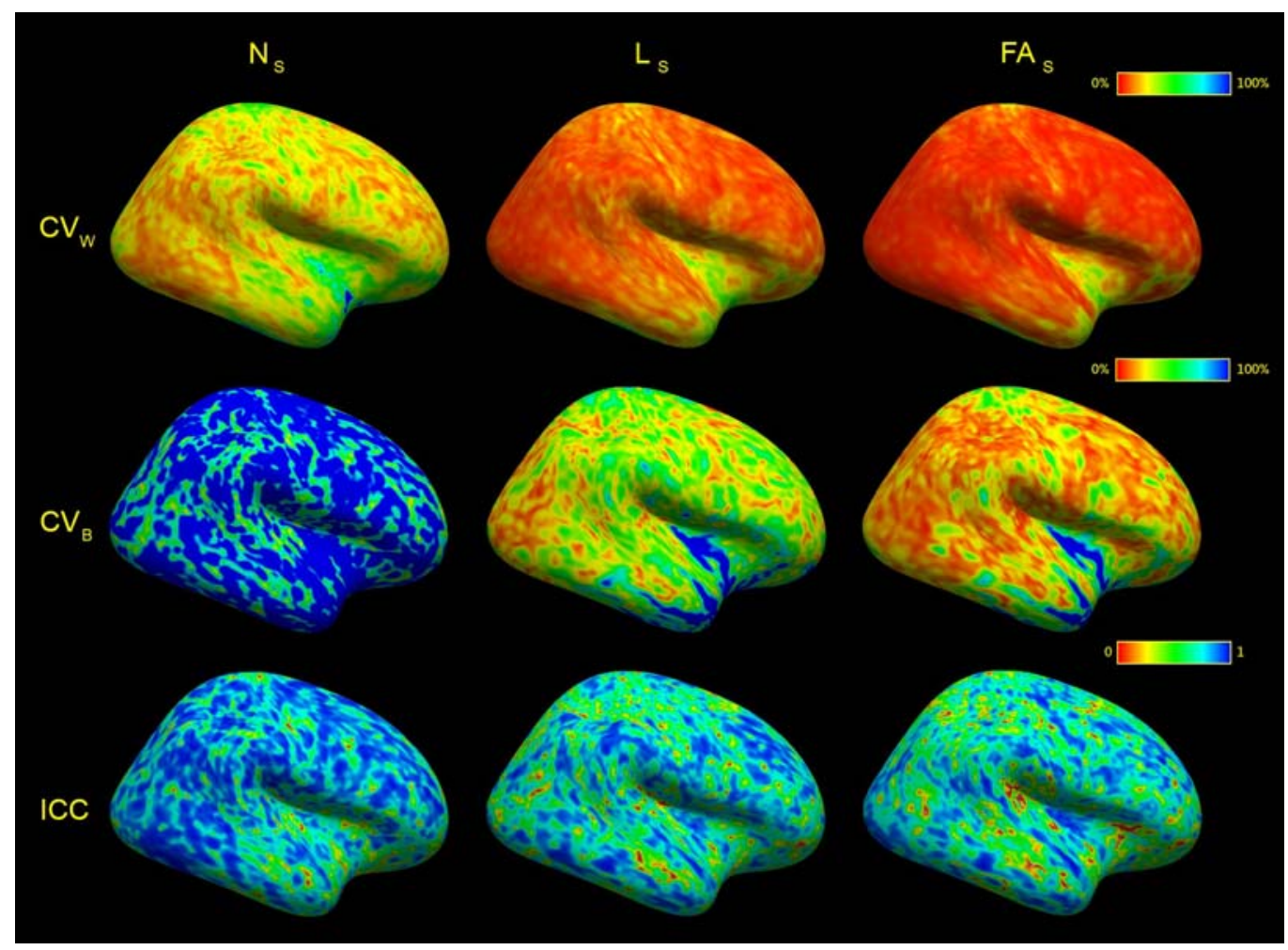

Figure 9: Surface-based analysis demonstrated on the lateral cortex of the right hemisphere. $\mathrm{N}_{\mathrm{S}}$, termination density (number of streamlines/vertex). $\mathrm{L}_{\mathrm{S}}$, mean streamline length/vertex. $\mathrm{FA}_{\mathrm{s}}$, mean streamline fractional anisotropy/vertex. $\mathrm{CV}_{\mathrm{W}}$, coefficient of 
variation within subjects. $\mathrm{CV}_{\mathrm{B}}$, coefficient of variation between subjects. ICC, intraclass correlation coefficient. Values at each vertex were recorded in subject space, then transformed into average subject space before running analyses. $\mathrm{CV}_{\mathrm{W}}$ and $\mathrm{CV}_{\mathrm{B}}$ were thresholded at $100 \%$.

$\mathrm{CV}_{\mathrm{B}}: 22.31 \%$ ). Fronto- and temporo-basal areas showed the least consistency, likely a result of susceptibility distortions arising from the EPIreadout used for the $\mathrm{dMRI}$ and $\mathrm{T} 1-\mathrm{dMRI}$ misalignment in those regions. Gyral bias appeared to also contribute to regional differences in consistency, with sulcal areas exhibiting higher reproducibility and reliability (Table 6).

\begin{tabular}{lrrr}
\hline Data on the surface $^{1}$ & CV $_{\text {w: gyri (\%) }}$ & $\mathbf{C V}_{\mathbf{w}}$ : sulci (\%) & \multicolumn{1}{c}{$\mathbf{p}_{2}$} \\
\hline Termination count after filtering: & $31.87 \pm 19.41$ & $30.97 \pm 18.12$ & 0.000 \\
Mean streamline length per vertex: & $16.43 \pm 15.25$ & $15.95 \pm 15.14$ & 0.000 \\
Mean streamline FA per vertex: & $11.56 \pm 13.14$ & $11.19 \pm 13.02$ & 0.000 \\
\hline
\end{tabular}

\begin{tabular}{lccc}
\hline Data on the surface $^{1}$ & $\mathbf{C V}_{\mathrm{B}}$ : gyri (\%) & $\mathbf{C V}_{\mathrm{B}}$ : sulci (\%) & p2 \\
\hline Termination count after filtering: & $106.92 \pm 42.87$ & $106.32 \pm 44.21$ & 0.000 \\
Mean streamline length per vertex: & $47.4 \pm 32.99$ & $46.47 \pm 34.21$ & 0.000 \\
Mean streamline FA per vertex: & $32.3 \pm 30.53$ & $31.88 \pm 32.17$ & 0.000 \\
\hline
\end{tabular}

\begin{tabular}{lllc}
\hline Data on the surface $^{1}$ & ICC: gyri & ICC: sulci & $\mathbf{p}^{2}$ \\
\hline Termination count after filtering: & $0.723 \pm 0.200$ & $0.728 \pm 0.195$ & 0.000 \\
Mean streamline length per vertex: & $0.666 \pm 0.205$ & $0.666 \pm 0.202$ & 0.913 \\
Mean streamline FA per vertex: & $0.641 \pm 0.209$ & $0.646 \pm 0.205$ & 0.000 \\
\hline 1 Definitions as in Table 5 & & & \\
2 Two-sample T-test (two-tailed) & & &
\end{tabular}

Table 6: Reproducibility of surface-projected streamline data in relation to cortical morphology and the effects of the gyral bias. Data are presented as mean \pm SD across all 
gyral or sulcal vertices. $\mathrm{CV}_{\mathrm{W}}$, coefficient of variation within subjects. $\mathrm{CV}_{\mathrm{B}}$, coefficient of variation between subjects. ICC, intraclass correlation coefficient.

\section{Discussion}

\subsection{Novelty of the work}

The work presented herein has offers a focused approach to short association fibre analysis by marrying tractography with mesh representation of the cortex motivated by the close association of SAF with the latter. We further utilised higher angular and/or spatial resolution as well as high b-values to improve the fidelity of the final tractograms. We were specifically interested in studying the shorter pathways (consistent with the definition in Schüz and Braitenberg (2002) as these pathways are particularly sensitive to inter-individual cortical folding variations (Bajada et al. (2019)) and harder to study using the more established approaches (Román et al. (2017); Zhang et al. (2018); Guevara et al. (2017); Van Essen et al. (2014)). We believe that this study adds a number of useful contributions to the literature.

First, the framework introduces simple yet strong anatomical constraints (three filters and a streamline length threshold) which operate in an unbiased fashion on a whole-brain level and require no assumptions regarding the shape or the distribution of these fibres on the cortical mantle. The resulting tractograms are consistent with the anatomical definition of Schüz and Braitenberg (2002) and require no manual pruning. The framework is modular and easily adaptable, enabling its use for studying SAF across a range of physiological and pathological conditions. It supports the use of a surface-based seeding approach as employed here but 
will also filter tractograms that were obtained with other means if desired. Using a bespoke algorithm, termination points of each streamline on the cortical mesh are registered during filtering, allowing direct interfacing between any streamline- and surface-related metrics (without reliance on the voxel grid) for subsequent analyses.

Second, we compare seeding strategies and the choice of scanner, acquisition, voxel size specifically in the context of SAF tractography. While the approach adopted here can be seen as resource-heavy, utilising ultrastrong gradient strengths with high-angular resolution dMRI, upsampling of data and the use of relatively time-consuming filters, through a series of experiments we demonstrate how this combination translates to betterdistributed, more anatomically plausible tractograms compared to a "standard" set-up.

Comparison between the Connectom/sa/ $1 \mathrm{~mm}^{3}$ and the MICRA acquisitions further suggested that despite having a lower spatial resolution, the latter produced tractograms of a similar if not superior quality. This is likely the result of a much higher angular resolution and better tissue discrimination. Higher angular resolution (Vos et al. (2016)), upsampling (Dyrby et al. (2014)), and surface-based approaches (Cottaar et al. (2021); St-Onge et al. (2018)) have all previously been demonstrated to improve tractography. We argue that the sensitivity of tracking in the subcortical areas to partial volume effects, gyral bias and complex fibre configurations in our opinion makes these adjustments even more worthwhile for our application. Although the Prisma/st data produced less satisfactory results, it is possible that image quality transfer techniques will allow to make use of the framework with the more "clinical" acquisitions 
enabling multicentre data collection in the future (Alexander et al. (2017); Tax et al. (2019)).

Third, we present a thorough evaluation of the tractograms generated with this method using state-of-the-art repeatability data (Koller et al. (2020)). The description process is broadly divided into streamline-, voxel-, and surface-wise assessments, to our knowledge representing the most complete characterisation of whole-brain SAF tractograms to date. This includes measures of fractional anisotropy chosen as an exemplar scalar to showcase performance of the pipeline for microstructure analysis; in future studies, a more exhaustive profiling of SAF microstructure may be conducted. The use of volume- and surface-based approaches allows to examine the differences in SAF properties on a regional level. While we did not utilise semi-global streamline optimisation algorithms (Smith et al. (2015); Daducci et al. (2015)), this still forms a useful baseline and informs future work.

Fourth, our approach to the representation of SAF metrics on the surface merits its own mention. Projection of streamline-related data on surface vertices is not new; however, usually (Padula et al. (2017); Bajada et al. (2019)) this has been achieved by searching for all streamlines within a (typically) large sphere around a WSM vertex which risks decreased specificity and leads to overlaps. In regions where non-continuous parts of the cortical mantle lie in close proximity with each other (such as the opposite banks of a narrow gyrus), erroneous inclusion of streamlines that terminate near remote vertices may occur. Alternatively, streamline density (Li et al. (2010); Nie et al. (2011)) and orientation termination (Chen et al. (2012)) around a surface vertex have been quantified as the number/orientation of streamlines penetrating the adjacent faces 
normalised by the combined surface area of the faces. While representing a more robust way to record these data on the surface, the latter approach allows for overlapping between adjacent vertices and, more importantly, on its own does not account for the streamlines not reaching the cortical mesh resulting in strong gyral bias. On the other hand, our method represents an improvement through the following combination: (1) allowing some propagation of streamlines into the cortex during seeding (later truncated at the interface), aided by higher spatial resolution and dMRI data upsampling, ensures more intersections of streamlines with the surface occur; (2) limiting the inclusion sphere to the local cortical thickness during filtering increases specificity of streamline-surface mapping; (3) subsequent fine-scale searching for intersections at the grey-white interface results in unique allocation of vertices based on proximity such that each streamline end is only associated with one vertex. This combination may lead to rejection of streamlines approaching but not entering the cortex (Yeh et al. (2019)); however, the proportion of such streamlines appeared negligible based on the distribution of rejected termination points (Appendix B) with an overall weak gyral bias (Table 5 and Table 6). Further, increased sensitivity could be achieved by expanding the WSM inwards during filtering using e.g. mean-curvature flow (St-Onge et al. (2018)), although this may lead to false positives in certain regions as, for example, the predominant orientation of axonal fibres around sulcal fundi is neartangential and thus the "passing" fibres may be erroneously included; hence this extra step was not pursued. Depending on the analyses of interest, subsequent smoothing on the surface may be applied as performed in this study. Further, projection of certain types of data (such as mean scalar 
measures along a streamline, e.g. fractional anisotropy) may not be justified in the context of whole-brain or deep-bundle tractography where many streamlines run at a distance from the cortex and exhibit vastly varying trajectories; on the other hand, this "collapsing" of data seems natural with SAF due to their short length and course that is inevitably local to the vertex to which data are being projected. Comparison of data represented in this way is achieved through surface registration which can handle cortical folding differences better than volume registration (critical when dealing with the immediate subcortical structures) with all other steps performed in native dMRI space. Finally, surface-based analysis provides the option of using per-vertex or cluster-based statistical comparison methods, circumventing the use of cortical parcellation if desired and therefore avoiding the associated issues of lower sensitivity within and artificial boundaries between cortical regions.

\subsection{Consistency of SAF tractograms}

Our analysis is complemented by a detailed evaluation of whole-brain SAF for consistency, including evaluation of reproducibility, reliability and between-subject variability. This demonstrated varying results depending on the approach taken. High overall reproducibility and reliability of streamline counts in the initial (not SAF-specific) tractograms dropped slightly as a result of filtering while still remaining within a good range. Streamline count can be influenced by a multitude of factors arising from processing, reconstruction and algorithmic choices but also anatomical variability, most of which can play a role at both the initial tractogram generation and filtering. Hence, on its own this may not be a good indicator 
of tractogram consistency. Instead, the number vertices with streamlines (cortical coverage) or number of streamlines per vertex (termination density) are possibly more insightful; in this work, reproducibility and reliability of both was high. On the other hand, mean streamline length demonstrated very low reliability. Here, the overall variance was so low that any within- and between-subject differences were likely at the noise level, suggesting that the whole-brain averaged length was not a useful measure for comparison. Indeed, examining streamline length at the vertex level yielded more informative results. As expected (Zhang et al. (2010)), track density imaging maps demonstrated large variability in the spatial distribution of SAF between individuals but also within individuals; in the attempt to minimise the role of registration imperfections and partial volume effects, alternative measures such as regional density and mean streamline length of SAF were compared by projecting them on the surface resulting in improved consistency. In addition to demonstrating the degree of consistency that could be expected for between-subject comparisons in studying physiological and pathological phenomena, the data offered here can be served as a baseline for future methodological developments. Our approach does not perform a dedicated removal of noisy (false positive) streamlines relying only on the anatomical constraints, and it is probable that a proportion of variability within and between subjects is explained by the occurrence of such streamlines owing to the fundamental limitations of a chosen tractography algorithm. Shape-based (Drakesmith et al. (2019); Parker et al. (2016)) exclusion of noisy streamlines could be undertaken in the future; however, the lack of detailed histological or tracer injection validation data for SAF on the whole-brain scale makes identification of 
such streamlines difficult. Development of better scanning hardware and tractography/optimisation algorithms will facilitate greater fidelity of the tractograms.

Consistency of whole-brain SAF representation with dMRI-based streamlines tractography approaches has previously been addressed. Zhang et al. (2010) used diffusion tensor imaging (DTI)-based deterministic tractography to create an atlas and a probabilistic spatial map of multiple white matter tracts, including all short association fibres connecting 24 regions obtained from a superficial white matter parcellation. Having detected 29 connections present in all 20 individuals studied, they emphasised large spatial variability (demonstrated but not quantified) and the difficulty in manual region-of-interest segmentation of these tracts advocating for an automated approach. In another study (Zhang et al. (2014)), shape-driven filtering and a further criterion of proximity to sulcal fundi were used to examine U-fibres across diffusion spectrum imaging (DSI), high angular resolution diffusion imaging (HARDI) and DTI data sets with deterministic streamlines tracking. Based on normalised streamline counts, the authors demonstrated an overall stronger short-range than middle-range connectivity with HARDI and DSI data and the reverse with DTI data, proposing the inability of the latter to detect crossing fibres and therefore more false negatives as the likely mechanism. Guevara et al. (2017) used DKT-based parcellation and shape- and distance-based clustering of larger streamlines (centroids $20-80 \mathrm{~mm}$ ) in Talairach space to identify 100 distinct bundles (50 per hemisphere, 35 common to both) that were considered to have low-to-moderate variability (relative standard deviation (RSD) $\leq 0.9$ ) in streamline counts and shape across two test and 
one validation data sets. This method was further improved by using nonlinear registration and ability to detect within-region connections (Román et al. (2017)); clustering larger (centroids 35-85 mm) streamlines and using a bagging strategy, the authors successfully constructed an atlas of $93 \mathrm{SAF}$ bundles (44 in left hemisphere, 49 in right, 33 common) with repeatability of individual bundles ranging between $8 / 10$ and 10/10.

Zhang et al. (2018) generated an atlas of white matter pathways based on 100 Human Connectome Project (HCP) subjects using two-tensor unscented Kalman filter for tracking and groupwise tractography registration followed by groupwise spectral clustering. Their approach did not require the use of a cortical parcellation. A total of 58 deep and 198 short and medium range superficial clusters were identified, although no further detailed description of the latter was provided. The classification was subsequently applied to a number of additional data sets with variable acquisition methods, spanning different age ranges and including clinical cohorts. Depending on the data set examined, this approach identified $92.28-99.96 \%$ of the "superficial" clusters on the subject level, with the average between-subject CV of 0.488-0.919 for streamline counts/cluster, and the average overlap between subject and atlas clusters of 0.747-0.783 (as determined using intersected FreeSurfer regions). Subsequent work (Guevara et al. (2020)) compared the three atlases (Guevara et al. (2017); Román et al. (2017); Zhang et al. (2018)) in MNI space for bundle similarity by computing the maximum Euclidean distance between corresponding points for each streamline in a bundle to all the streamlines in another bundle. With cut-offs for distance and percentage of similar streamlines of 8 $\mathrm{mm}$ and $\leq 80 \%$, respectively, there was a good overlap between the 
bundles, particularly in frontal and parietal areas, the atlas of Zhang et al. (2018) contained 96 bundles not present in the other two atlases. The same paper compared the impact of different tractography algorithms (DTI, generalized Q-sampling imaging or GQI and MRtrix iF0D2 using ACT+SIFT) on consistency of clustering, showing that the probabilistic tracking with MRtrix was able to reconstruct all bundles in $100 \%$ of cases with a greater spatial coverage but with a higher streamline count RSD compared to the other algorithms. As mentioned previously, these streamline clustering approaches appear to show a good performance when classifying the larger cortical connections but are not typically applied to the smaller ones. While not studying SAF on a whole-brain scale, the study by Movahedian Attar et al. (2020) is of special interest as similarly to our work it relied on the length definition of Schüz and Braitenberg (2002) and used dMRI data acquired using ultra-high gradient Connectom scanner (choosing higher spatial resolution over higher b-values). The study evaluated connectivity (using relative streamline counts) within the occipital cortex as defined by fMRI regions-of-interest and demonstrated a test-retest ICC of $0.73 \pm 0.33$ $(0.88 \pm 0.70$ for "retinotopic" and $0.69 \pm 0.35$ for "non-retinotopic", considered false positive by design, bundles) and an averaged CoV of 0.23 $(0.23 \pm 0.23$ for "retinotopic" and $0.25 \pm 0.14$ for "non-retinotopic" bundles). The use of multimodal surface registration algorithms allowing integration of structural and functional units of the cortex (Robinson et al. (2014)) resulting in similar assessments on a whole-brain scale is a future interest to extend our work. 


\subsection{Limitations}

Some limitations of the proposed approach should be mentioned. First, the dMRI repeatability data used in this study had a voxel size of $2 \times 2 \times 2$ $\mathrm{mm}^{3}$. While previous work has suggested the ideal voxel size below 0.85 mm (isotropic) to remain sensitive to the smaller component of SAF (Song et al. (2014); Movahedian Attar et al. (2020)), the voxel size used in our cohort is representative of what is commonly used in diffusion studies; further, our data were acquired with higher b-values and using high angular resolution, limiting partial volume effects, increasing sensitivity to the intraaxonal component of the white matter thus allowing a better resolution of complex fibre orientations (Novikov et al. (2019); Vos et al. (2016); Jeurissen et al. (2014)) while maintaining a good signal-to-noise ratio due to the use of high gradient strength (Jones et al. (2018)). We also upsampled our data as it has been shown to improve the geometrical representation of white matter tracts

(Dyrby et al. (2014); Shastin et al. (2019)). The second limitation is the sensitivity of the framework to registration quality between T1-weighted and dMRI data. Data sets containing distortions (such as susceptibility artifact) or unusual anatomy (e.g., tumours) are likely to have a mismatch between the surfaces reconstructed from T1-weighted images and the white matter signal on dMRI. As such, visual inspection is crucial on an individual basis although we did not encounter any issues with registration. dMRI-based surface extraction could offer an alternative solution (Liu et al. (2007); Li et al. (2010); Shastin et al. (2020)) if performed at sufficiently high resolution. Third, the current approach to surface seeding precludes the use of semiglobal streamline optimisation algorithms (Smith et al. 
(2015); Daducci et al. (2015)) which require whole-brain tractograms. This limitation can be easily overcome by including the remaining grey matter structures (subcortical grey, cerebellar cortex, amygdalae, and hippocampi) into the array of seeding coordinates and applying the optimisation algorithms before subsequent filtering, preserving streamline weights throughout the process. Fourth, as mentioned previously, this paper used the length definition of SAF as under $40 \mathrm{~mm}$ minus the variable intracortical section (mean 19.11 $\pm 0.14 \mathrm{~mm}$ ). While application of our method without this additional criterion resulted in a similar (albeit denser) tractogram appearances and was dominated by short streamlines (mean $\approx 30 \mathrm{~mm}$, data not shown, consistent with Padula et al. (2017)), it could be similarly used to study larger subcortical association fibres although extra filters such as passage in the vicinity of sulci (Zhang et al. (2014)) may be needed. Finally, the pipeline took about 4 hours to run using parallel CPUs. This computational cost may be less appropriate for long range white matter tractography, where exact interaction between streamlines and cortical structures may be less crucial. However, based on our experiments we judged that such approach was suitable for SAF tractography. Further developments to the framework could include GPU-based execution and the use geodesic distance rather than K-means for cortical coordinate clustering.

\section{Conclusions}

We consider our work to be a first-time application of a dedicated whole-brain, surface-based SAF tractography approach aimed at overcoming some of the limitations pertinent to the study of this white 
matter population. Surface-based seeding employed here was demonstrated to result in a more uniform subcortical streamline distribution. Surfacebased filtering algorithms coupled with a streamline length criterion $(\leq 30$ $40 \mathrm{~mm}$ ) were used to produce tractograms with a large cortical coverage and appeared to be well-distributed in the superficial regions of white matter. Higher gradient strengths and higher angular resolution were demonstrated to produce fODFs that better penetrated the grey-white interface and had greater magnitudes near the gyral crowns. Together with resampling of dMRI data, these factors have been associated with tractograms that had higher streamline counts, greater cortical coverage, a slightly improved mean angle between the cortex and the streamlines, but also relatively more streamlines connecting gyral crowns and the adjacent walls. We characterised SAF and assessed their consistency using a variety of complimentary approaches, presenting the framework as a vehicle for investigating SAF in health as well as in clinical cohorts and offering benchmark data for future methodological improvements.

Declarations of interest: none

\section{Author contributions}

Dmitri Shastin: conceptualization, methodology, software, validation, formal analysis, writing - original draft, writing - review \& editing, visualization, funding acquisition. Sila Genc: methodology, software, writing original draft. Greg D. Parker: conceptualization, software. Kristin Koller: resources, data curation. Chantal M.W. Tax: resources, data curation, methodology, writing - review \& editing. John Evans: resources, 
methodology. Khalid Hamandi: writing - review \& editing, supervision. William P. Gray: writing - review \& editing, conceptualization, supervision. Derek K. Jones: conceptualization, writing - original draft, writing - review \& editing, supervision. Maxime Chamberland: conceptualization, writing original draft, writing - review \& editing, visualization, supervision.

\section{Acknowledgements}

This research was funded in whole, or in part, by a Wellcome Trust Investigator Award (096646/Z/11/Z), a Wellcome Trust Strategic Award (104943/Z/14/Z), a Wellcome Trust-funded GW4-CAT fellowship (215944/Z/19/Z), the Engineering and Physical Sciences Research Council (EP/M029778/1), the Dutch Research Council (17331), a Radboud Excellence Initiative Fellowship, and the Brain Repair and Intracranial Neurotherapeutics (BRAIN) Unit funded by Health and Care Research Wales. For the purpose of open access, the author has applied a CC BY public copyright licence to any Author Accepted Manuscript version arising from this submission.

DS would like to thank Prof. Peter H. Morgan at Cardiff Business School for discussions about statistics. 


\section{References}

Alexander, D.C., Zikic, D., Ghosh, A., Tanno, R., Wottschel, V., Zhang, J., Kaden, E., Dyrby, T.B., Sotiropoulos, S.N., Zhang, H., Criminisi, A., 2017. Image quality transfer and applications in diffusion mri. Neuroimage 152, 283298. doi:10.1016/j.neuroimage.2017.02.089.

Andersson, J.L., Skare, S., Ashburner, J., 2003. How to correct susceptibility distortions in spin-echo echo-planar images: application to diffusion tensor imaging. Neuroimage 20, 870-88. doi:10.1016/S10538119(03)00336-7.

Andersson, J.L.R., Sotiropoulos, S.N., 2016. An integrated approach to correction for off-resonance effects and subject movement in diffusion MR imaging. Neuroimage 125, 1063-1078. doi:10.1016/j.neuroimage.2015.10. 019.

Arthur, D., Vassilvitskii, S., 2006. k-means++: The Advantages of Careful Seeding. techreport. Stanford InfoLab.

Avants, B.B., Tustison, N., Song, G., 2009. Advanced normalization tools (ants). Insight j 2, 1-35.

Bajada, C.J., Schreiber, J., Caspers, S., 2019. Fiber length profiling: A novel approach to structural brain organization. Neuroimage 186, 164-173.

Calamante, F., Tournier, J.D., Jackson, G.D., Connelly, A., 2010. Track density imaging (TDI): super-resolution white matter imaging using whole-brain track-density mapping. Neuroimage 53, 1233-1243. 
Chen, G., Taylor, P.A., Haller, S.P., Kircanski, K., Stoddard, J., Pine, D.S., Leibenluft, E., Brotman, M.A., Cox, R.W., 2018. Intraclass correlation: Improved modeling approaches and applications for neuroimaging. Human brain mapping 39, 1187-1206.

Chen, H., Zhang, T., Guo, L., Li, K., Yu, X., Li, L., Hu, X., Han, J., Hu, X., Liu, T., 2012. Coevolution of Gyral Folding and Structural Connection Patterns in Primate Brains. Cerebral Cortex 23, 1208-1217. doi:10.1093/ cercor/bhs113.

Cottaar, M., Bastiani, M., Boddu, N., Glasser, M.F., Haber, S., Van Essen, D.C., Sotiropoulos, S.N., Jbabdi, S., 2021. Modelling white matter in gyral blades as a continuous vector field. NeuroImage 227, 117693.

Daducci, A., Dal Palu, A., Lemkaddem, A., Thiran, J.P., 2015. Commit: Convex optimization modeling for microstructure informed tractography. IEEE Trans Med Imaging 34, 246-57. doi:10.1109/TMI.2014.2352414.

Datta, G., Colasanti, A., Rabiner, E.A., Gunn, R.N., Malik, 0., Ciccarelli, 0., Nicholas, R., Van Vlierberghe, E., Van Hecke, W., Searle, G., et al., 2017. Neuroinflammation and its relationship to changes in brain volume and white matter lesions in multiple sclerosis. Brain 140, 2927-2938.

Dhollander, T., Raffelt, D., Connelly, A., 2016. Unsupervised 3-tissue response function estimation from single-shell or multi-shell diffusion $\mathrm{mr}$ data without a co-registered t1 image, in: ISMRM Workshop on Breaking the Barriers of Diffusion MRI, p. 5.

Drakesmith, M., Parker, G.D., Smith, J., Linden, S.C., Rees, E., Williams, N., Owen, M.J., van den Bree, M., Hall, J., Jones, D.K., Linden, D.E.J., 2019. 
Genetic risk for schizophrenia and developmental delay is associated with shape and microstructure of midline white-matter structures. Transl Psychiatry 9, 102. doi:10.1038/s41398-019-0440-7.

Dubois, J., Dehaene-Lambertz, G., Kulikova, S., Poupon, C., Hüppi, P.S., HertzPannier, L., 2014. The early development of brain white matter: a review of imaging studies in fetuses, newborns and infants. Neuroscience 276, 48-71.

Dyrby, T.B., Lundell, H., Burke, M.W., Reislev, N.L., Paulson, O.B., Ptito, M., Siebner, H.R., 2014. Interpolation of diffusion weighted imaging datasets. Neuroimage 103, 202-213. doi:10.1016/j.neuroimage.2014. 09.005.

d'Albis, M.A., Guevara, P., Guevara, M., Laidi, C., Boisgontier, J., Sarrazin, S., Duclap, D., Delorme, R., Bolognani, F., Czech, C., et al., 2018. Local structural connectivity is associated with social cognition in autism spectrum disorder. Brain 141, 3472-3481.

Fischl, B., 2012. Freesurfer. Neuroimage 62, 774-781.

Fischl, B., Sereno, M.I., Tootell, R.B., Dale, A.M., 1999. High-resolution intersubject averaging and a coordinate system for the cortical surface. Hum Brain Mapp 8, 272-84. doi:10.1002/(sici)1097-0193(1999)8:4<272:: aid-hbm10>3.0.co;2-4.

Genc, S., Tax, C.M., Raven, E.P., Chamberland, M., Parker, G.D., Jones, D.K., 2020. Impact of b-value on estimates of apparent fibre density. Human brain mapping 41, 2583-2595. 
Glasser, M.F., Sotiropoulos, S.N., Wilson, J.A., Coalson, T.S., Fischl, B., Andersson, J.L., Xu, J., Jbabdi, S., Webster, M., Polimeni, J.R., Van Essen, D.C., Jenkinson, M., Consortium, W.U.M.H., 2013. The minimal preprocessing pipelines for the human connectome project. Neuroimage 80, 105-24. doi:10.1016/j.neuroimage.2013.04.127.

Govindan, R.M., Asano, E., Juhasz, C., Jeong, J.W., Chugani, H.T., 2013. Surfacebased laminar analysis of diffusion abnormalities in cortical and white matter layers in neocortical epilepsy. Epilepsia 54, 667-77. doi:10. 1111/epi.12129.

de Groot, M., Ikram, M.A., Akoudad, S., Krestin, G.P., Hofman, A., van der Lugt, A., Niessen, W.J., Vernooij, M.W., 2015. Tract-specific white matter degeneration in aging: the rotterdam study. Alzheimer's \& Dementia 11, $321-330$.

Guevara, M., Guevara, P., Roman, C., Mangin, J.F., 2020. Superficial white matter: A review on the dmri analysis methods and applications. Neuroimage 212, 116673. doi:10.1016/j.neuroimage.2020.116673.

Guevara, M., Roman, C., Houenou, J., Duclap, D., Poupon, C., Mangin, J.F., Guevara, P., 2017. Reproducibility of superficial white matter tracts using diffusion-weighted imaging tractography. Neuroimage 147, 703-725. doi:10.1016/j.neuroimage.2016.11.066.

Hihara, S., Notoya, T., Tanaka, M., Ichinose, S., Ojima, H., Obayashi, S., Fujii, N., Iriki, A., 2006. Extension of corticocortical afferents into the anterior bank of the intraparietal sulcus by tool-use training in adult monkeys. Neuropsychologia 44, 2636-2646. 
Jeurissen, B., Descoteaux, M., Mori, S., Leemans, A., 2017. Diffusion mri fiber tractography of the brain. NMR Biomed doi:10.1002/nbm.3785.

Jeurissen, B., Tournier, J.D., Dhollander, T., Connelly, A., Sijbers, J., 2014. Multi-tissue constrained spherical deconvolution for improved analysis of multi-shell diffusion mri data. Neuroimage 103, 411-426. doi:10.1016/j. neuroimage.2014.07.061.

Jones, D.K., Alexander, D.C., Bowtell, R., Cercignani, M., Dell'Acqua, F., McHugh, D.J., Miller, K.L., Palombo, M., Parker, G.J.M., Rudrapatna, U.S., Tax, C.M.W., 2018. Microstructural imaging of the human brain with a 'super-scanner': 10 key advantages of ultra-strong gradients for diffusion MRI. Neuroimage 182, 8-38. doi:10.1016/j.neuroimage.2018.05.047.

Jones, D.K., Symms, M.R., Cercignani, M., Howard, R.J., 2005. The effect of filter size on vbm analyses of dt-mri data. Neuroimage 26, 546-554.

Kai, J., Khan, A.R., 2019. Assessing white matter pathway reproducibility from human whole-brain tractography clustering. bioRxiv .

Kellner, E., Dhital, B., Kiselev, V.G., Reisert, M., 2016. Gibbs-ringing artifact removal based on local subvoxel-shifts. Magn Reson Med 76, 1574-1581. doi:10.1002/mrm.26054.

Koller, K., Rudrapatna, S.U., Chamberland, M., Raven, E.P., Parker, G.D., Tax, C.M., Drakesmith, M., Fasan, F., Owen, D., Hughes, G., et al., 2020. Micra: Microstructural image compilation with repeated acquisitions. NeuroImage , 117406.

Laguna, P.A.L., Combes, A.J., Streffer, J., Einstein, S., Timmers, M., Williams, S.C., Dell'Acqua, F., 2020. Reproducibility, reliability and variability of FA 
and md in the older healthy population: A test-retest multiparametric analysis. NeuroImage: Clinical 26.

Li, K., Guo, L., Li, G., Nie, J., Faraco, C., Cui, G., Zhao, Q., Miller, L.S., Liu, T., 2010. Gyral folding pattern analysis via surface profiling. NeuroImage 52 , $1202-1214$.

Liu, M., Bernhardt, B.C., Hong, S.J., Caldairou, B., Bernasconi, A., Bernasconi, N., 2016. The superficial white matter in temporal lobe epilepsy: a key link between structural and functional network disruptions. Brain 139, 2431-40. doi:10.1093/brain/aww167.

Liu, T., Li, H., Wong, K., Tarokh, A., Guo, L., Wong, S.T., 2007. Brain tissue segmentation based on dti data. NeuroImage 38, 114 - 123. doi: https: //doi.org/10.1016/j.neuroimage.2007.07.002.

Maier-Hein, K.H., Neher, P.F., Houde, J.C., Cote, M.A., Garyfallidis, E., Zhong, J., Chamberland, M., Yeh, F.C., Lin, Y.C., Ji, Q., Reddick, W.E., Glass, J.O., Chen, D.Q., Feng, Y., Gao, C., Wu, Y., Ma, J., He, R., Li, Q., Westin, C.F., DeslauriersGauthier, S., Gonzalez, J.0.0., Paquette, M., St-Jean, S., Girard, G., Rheault, F., Sidhu, J., Tax, C.M.W., Guo, F., Mesri, H.Y., David, S., Froeling, M., Heemskerk, A.M., Leemans, A., Bore, A., Pinsard, B., Bedetti, C., Desrosiers, M., Brambati,

S., Doyon, J., Sarica, A., Vasta, R., Cerasa, A., Quattrone, A., Yeatman, J., Khan, A.R., Hodges, W., Alexander, S., Romascano, D., Barakovic, M., Auria, A., Esteban, 0., Lemkaddem, A., Thiran, J.P., Cetingul, H.E., Odry, B.L., Mailhe, B., Nadar, M.S., Pizzagalli, F., Prasad, G., Villalon-Reina, J.E., Galvis, J., Thompson, P.M., Requejo, F.S., Laguna, P.L., Lacerda, L.M., Barrett, R., 
Dell'Acqua, F., Catani, M., Petit, L., Caruyer, E., Daducci, A., Dyrby, T.B., Holland-Letz, T., Hilgetag, C.C., Stieltjes, B., Descoteaux, M., 2017. The challenge of mapping the human connectome based on diffusion tractography. Nat Commun 8, 1349. doi:10.1038/s41467-017-01285-x.

Markov, N.T., Ercsey-Ravasz, M., Ribeiro Gomes, A., Lamy, C., Magrou, L., Vezoli, J., Misery, P., Falchier, A., Quilodran, R., Gariel, M., et al., 2014. A weighted and directed interareal connectivity matrix for macaque cerebral cortex. Cerebral cortex 24, 17-36.

McGraw, K.0., Wong, S.P., 1996. Forming inferences about some intraclass correlation coefficients. Psychological methods 1, 30.

Mito, R., Raffelt, D., Dhollander, T., Vaughan, D.N., Tournier, J.D., Salvado, O., Brodtmann, A., Rowe, C.C., Villemagne, V.L., Connelly, A., 2018. Fibrespecific white matter reductions in alzheimer's disease and mild cognitive impairment. Brain 141, 888-902.

Mori, S., Van Zijl, P.C., 2002. Fiber tracking: principles and strategies-a technical review. NMR in Biomedicine: An International Journal Devoted to the Development and Application of Magnetic Resonance In Vivo 15, 468-480.

Movahedian Attar, F., Kirilina, E., Haenelt, D., Pine, K.J., Trampel, R., Edwards, L.J., Weiskopf, N., 2020. Mapping short association fibers in the early cortical visual processing stream using in vivo diffusion tractography. Cereb Cortex doi:10.1093/cercor/bhaa049.

Nazeri, A., Chakravarty, M.M., Felsky, D., Lobaugh, N.J., Rajji, T.K., Mulsant, B.H., Voineskos, A.N., 2013. Alterations of superficial white matter in 
schizophrenia and relationship to cognitive performance. Neuropsychopharmacology 38, 1954-1962.

Neubert, F.X., Mars, R.B., Buch, E.R., Olivier, E., Rushworth, M.F.S., 2010. Cortical and subcortical interactions during action reprogramming and their related white matter pathways. Proceedings of the National Academy of Sciences 107, 13240-13245. doi:10.1073/pnas.1000674107.

Nie, J., Guo, L., Li, K., Wang, Y., Chen, G., Li, L., Chen, H., Deng, F., Jiang, X., Zhang, T., Huang, L., Faraco, C., Zhang, D., Guo, C., Yap, P.T., Hu, X., Li, G., Lv, J., Yuan, Y., Zhu, D., Han, J., Sabatinelli, D., Zhao, Q., Miller, L.S., Xu, B., Shen, P., Platt, S., Shen, D., Hu, X., Liu, T., 2011. Axonal Fiber Terminations Concentrate on Gyri. Cerebral Cortex 22, 2831-2839. doi:10.1093/cercor/bhr361.

Novikov, D.S., Fieremans, E., Jespersen, S.N., Kiselev, V.G., 2019. Quantifying brain microstructure with diffusion mri: Theory and parameter estimation. NMR in Biomedicine 32, e3998.

O’Halloran, R., Feldman, R., Marcuse, L., Fields, M., Delman, B., Frangou, S., Balchandani, P., 2017. A method for u-fiber quantification from $7 \mathrm{t}$ diffusion-weighted mri data tested in patients with nonlesional focal epilepsy. Neuroreport 28, 457-461. doi:10.1097/WNR.0000000000000788.

Ouyang, M., Kang, H., Detre, J.A., Roberts, T.P., Huang, H., 2017. Shortrange connections in the developmental connectome during typical and atypical brain maturation. Neuroscience \& Biobehavioral Reviews 83, 109- 122. 
Padula, M.C., Schaer, M., Scariati, E., Mutlu, A.K., Zo"ller, D., Schneider, M., Eliez, S., 2017. Quantifying indices of short-and long-range white matter connectivity at each cortical vertex. PloS one 12, e0187493.

Parker, G.D., Evans, G.J., Jones, D.K., 2016. A novel framework for global comparison of tract-topology between subjects reveals callosum shape variations in first episode psychosis, in: Proc. of the International Society for Magnetic Resonance in Medicine, p. 4131.

Phillips, O.R., Clark, K.A., Luders, E., Azhir, R., Joshi, S.H., Woods, R.P., Mazziotta, J.C., Toga, A.W., Narr, K.L., 2013. Superficial white matter: effects of age, sex, and hemisphere. Brain Connect 3, 146-59. doi:10. 1089/brain.2012.0111.

Phillips, O.R., Joshi, S.H., Narr, K.L., Shattuck, D.W., Singh, M., Di Paola, M., Ploner, C.J., Pruss, H., Paul, F., Finke, C., 2018. Superficial white matter damage in anti-nmda receptor encephalitis. J Neurol Neurosurg Psychiatry 89, 518-525. doi:10.1136/jnnp-2017-316822.

Phillips, O.R., Nuechterlein, K.H., Asarnow, R.F., Clark, K.A., Cabeen, R., Yang, Y., Woods, R.P., Toga, A.W., Narr, K.L., 2011. Mapping corticocortical structural integrity in schizophrenia and effects of genetic liability. Biol Psychiatry 70, 680-9. doi:10.1016/j.biopsych.2011.03.039.

Pierpaoli, C., Jezzard, P., Basser, P.J., Barnett, A., Di Chiro, G., 1996. Diffusion tensor mr imaging of the human brain. Radiology 201, 637-648.

Rademacher, J., 2002. Topographical variability of cytoarchitectonic areas, in: Cortical Areas. CRC Press, pp. 65-90. 
Reuter, M., Schmansky, N.J., Rosas, H.D., Fischl, B., 2012. Within-subject template estimation for unbiased longitudinal image analysis. Neuroimage 61, 1402-1418.

Reveley, C., Seth, A.K., Pierpaoli, C., Silva, A.C., Yu, D., Saunders, R.C., Leopold, D.A., Ye, F.Q., 2015. Superficial white matter fiber systems impede detection of long-range cortical connections in diffusion mr tractography. Proc Natl Acad Sci U S A 112, E2820-8. doi:10.1073/pnas. 1418198112.

Rheault, F., Poulin, P., Valcourt Caron, A., St-Onge, E., Descoteaux, M., 2020. Common misconceptions, hidden biases and modern challenges of dmri tractography. J Neural Eng doi:10.1088/1741-2552/ab6aad.

Robinson, E.C., Jbabdi, S., Glasser, M.F., Andersson, J., Burgess, G.C., Harms, M.P., Smith, S.M., Van Essen, D.C., Jenkinson, M., 2014. Msm: a new flexible framework for multimodal surface matching. Neuroimage 100, 414-426.

Román, C., Guevara, M., Valenzuela, R., Figueroa, M., Houenou, J., Duclap, D., Poupon, C., Mangin, J.F., Guevara, P., 2017. Clustering of wholebrain white matter short association bundles using hardi data. Frontiers in neuroinformatics 11,73 .

Rudrapatna, S.U., Parker, G.D., Roberts, J., Jones, D.K., 2018. Can we correct for interactions between subject motion and gradient-nonlinearity in diffusion mri, in: Proc. Int. Soc. Mag. Reson. Med.

Sairanen, V., Leemans, A., Tax, C.M.W., 2018. Fast and accurate slicewise outlier detection (solid) with informed model estimation for diffusion mri data. Neuroimage 181, 331-346. doi:10.1016/j.neuroimage.2018.07. 003. 
de Schipper, L.J., Hafkemeijer, A., Bouts, M.J., van der Grond, J., Marinus, J.,

Henselmans, J.M., van Hilten, J.J., 2019. Age-and disease-related cerebral white matter changes in patients with parkinson's disease. Neurobiology of aging $80,203-209$.

Schmahmann, J., Pandya, D., 2006. Fiber pathways of the brain oxford university press. New York.

Scholz, J., Klein, M.C., Behrens, T.E., Johansen-Berg, H., 2009. Training induces changes in white-matter architecture. Nature neuroscience 12, $1370-1371$.

Schüz, A., Braitenberg, V., 2002. The human cortical white matter: quantitative aspects of cortico-cortical long-range connectivity. Cortical areas: Unity and diversity , 377-385.

Setsompop, K., Kimmlingen, R., Eberlein, E., Witzel, T., Cohen-Adad, J., McNab, J.A., Keil, B., Tisdall, M.D., Hoecht, P., Dietz, P., Cauley, S.F., Tountcheva, V., Matschl, V., Lenz, V.H., Heberlein, K., Potthast, A., Thein, H., Van Horn, J., Toga, A., Schmitt, F., Lehne, D., Rosen, B.R., Wedeen, V., Wald, L.L., 2013. Pushing the limits of in vivo diffusion mri for the human connectome project. Neuroimage 80, 220-33. doi:10.1016/j.neuroimage.2013.05.078.

Shastin, D., Chamberland, M., Parker, G., Tax, C.M.W., Koller, K., Hamandi, K., Gray, W., Jones, D., 2020. Delineating the grey matterwhite matter interface directly from diffusion mri data, in: ISMRM, p. 4385. 
Shastin, D., Rudrapatna, S.U., Parker, G., Hamandi, K., Gray, W., Jones, D., Chamberland, M., 2019. Tractography of complex white matter bundles: limitations of diffusion mri data upsampling, in: ISMRM, p. 3318.

Slater, D.A., Melie-Garcia, L., Preisig, M., Kherif, F., Lutti, A., Draganski, B., 2019. Evolution of white matter tract microstructure across the life span. Human brain mapping 40, 2252-2268.

Smith, R.E., Tournier, J.D., Calamante, F., Connelly, A., 2012. Anatomicallyconstrained tractography: improved diffusion mri streamlines tractography through effective use of anatomical information. Neuroimage 62, 1924-38. doi:10.1016/j.neuroimage.2012.06.005.

Smith, R.E., Tournier, J.D., Calamante, F., Connelly, A., 2015. Sift2: Enabling dense quantitative assessment of brain white matter connectivity using streamlines tractography. Neuroimage 119, 338-351.

Song, A.W., Chang, H.C., Petty, C., Guidon, A., Chen, N.K., 2014. Improved delineation of short cortical association fibers and gray/white matter boundary using whole-brain three-dimensional diffusion tensor imaging at submillimeter spatial resolution. Brain Connect 4, 636-40. doi:10.1089/brain.2014.0270.

St-Onge, E., Daducci, A., Girard, G., Descoteaux, M., 2018. Surface-enhanced tractography (set). Neuroimage 169, 524-539. doi:10.1016/j.neuroimage.2017.12.036.

Steinmetz, H., Fu"rst, G., Freund, H.J., 1989. Cerebral cortical localization: application and validation of the proportional grid system in $\mathrm{mr}$ imaging. Journal of computer assisted tomography 13, 10-19. 
Stejskal, E.0., Tanner, J.E., 1965. Spin diffusion measurements: spin echoes in the presence of a time-dependent field gradient. The journal of chemical physics $42,288-292$.

Tax, C.M., Grussu, F., Kaden, E., Ning, L., Rudrapatna, U., Evans, C.J., St-Jean, S., Leemans, A., Koppers, S., Merhof, D., et al., 2019. Crossscanner and cross-protocol diffusion mri data harmonisation: A benchmark database and evaluation of algorithms. NeuroImage 195, 285-299.

Tournier, J.D., Calamante, F., Connelly, A., 2007. Robust determination of the fibre orientation distribution in diffusion mri: non-negativity constrained super-resolved spherical deconvolution. Neuroimage 35, 1459-72. doi:10.1016/j.neuroimage.2007.02.016.

Tournier, J.D., Calamante, F., Connelly, A., 2010. Improved probabilistic streamlines tractography by 2 nd order integration over fibre orientation distributions, in: Proceedings of the international society for magnetic resonance in medicine, Ismrm.

Tournier, J.D., Smith, R., Raffelt, D., Tabbara, R., Dhollander, T., Pietsch, M., Christiaens, D., Jeurissen, B., Yeh, C.H., Connelly, A., 2019. Mrtrix3: A fast, flexible and open software framework for medical image processing and visualisation. NeuroImage , 116-137.

Van Essen, D.C., Jbabdi, S., Sotiropoulos, S.N., Chen, C., Dikranian, K., Coalson, T., Harwell, J., Behrens, T.E., Glasser, M.F., 2014. Mapping connections in humans and non-human primates: aspirations and challenges for diffusion imaging, in: Diffusion MRI. Elsevier, pp. 337-358. 
Vos, S.B., Aksoy, M., Han, Z., Holdsworth, S.J., Maclaren, J., Viergever, M.A., Leemans, A., Bammer, R., 2016. Trade-off between angular and spatial resolutions in in vivo fiber tractography. Neuroimage 129, 117-132. doi:10.1016/j.neuroimage.2016.01.011.

Vos, S.B., Tax, C.M., Luijten, P.R., Ourselin, S., Leemans, A., Froeling, M., 2017. The importance of correcting for signal drift in diffusion mri. Magn Reson Med 77, 285-299. doi:10.1002/mrm.26124.

Yeh, C.H., Smith, R.E., Dhollander, T., Calamante, F., Connelly, A., 2019. Connectomes from streamlines tractography: Assigning streamlines to brain parcellations is not trivial but highly consequential. Neuroimage $199,160-171$.

Zhang, F., Wu, Y., Norton, I., Rigolo, L., Rathi, Y., Makris, N., O’Donnell, L.J., 2018. An anatomically curated fiber clustering white matter atlas for consistent white matter tract parcellation across the lifespan. NeuroImage 179, 429-447.

Zhang, T., Chen, H., Guo, L., Li, K., Li, L., Zhang, S., Shen, D., Hu, X., Liu, T., 2014. Characterization of $\mathrm{u}$-shape streamline fibers: Methods and applications. Medical image analysis 18, 795-807.

Zhang, Y., Zhang, J., Oishi, K., Faria, A.V., Jiang, H., Li, X., Akhter, K., Rosa-Neto, P., Pike, G.B., Evans, A., et al., 2010. Atlas-guided tract reconstruction for automated and comprehensive examination of the white matter anatomy. Neuroimage 52, 1289-1301. 


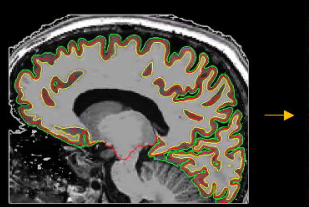

surface extraction

(Freesurfer 7.1)

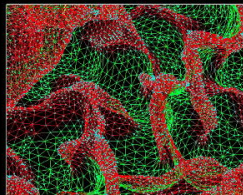

white surface vertex coordinates $\rightarrow \mathrm{dMR}$ space

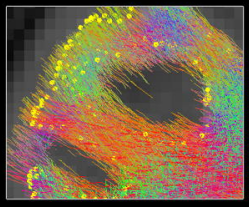

iFOD2 tracking from

vertices as seeds

(MRtrix3.0, modified)

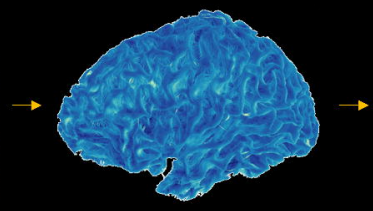

tractogram filtering

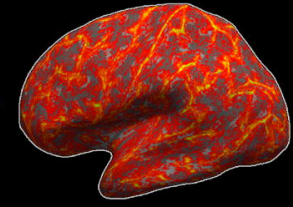

data projection on surfaces and analysis

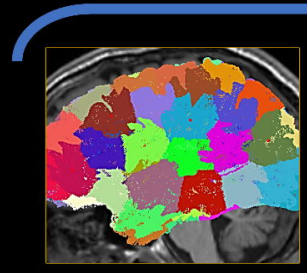

surface vertex grouping using $\mathrm{K}$-means

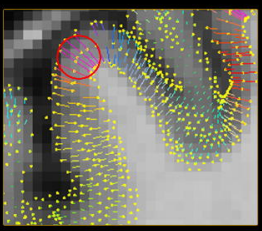

formation of mid-cortical coordinates, calculation of local cortical half-thickness

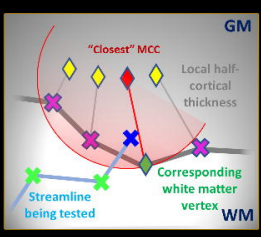

association of each streamline with its closest MCC (both ends)

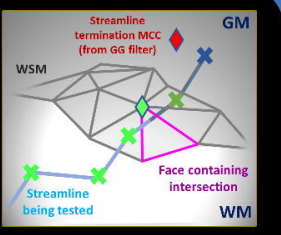

streamline-white surface intersection detection (both ends)

\section{GG and HH filters}


B
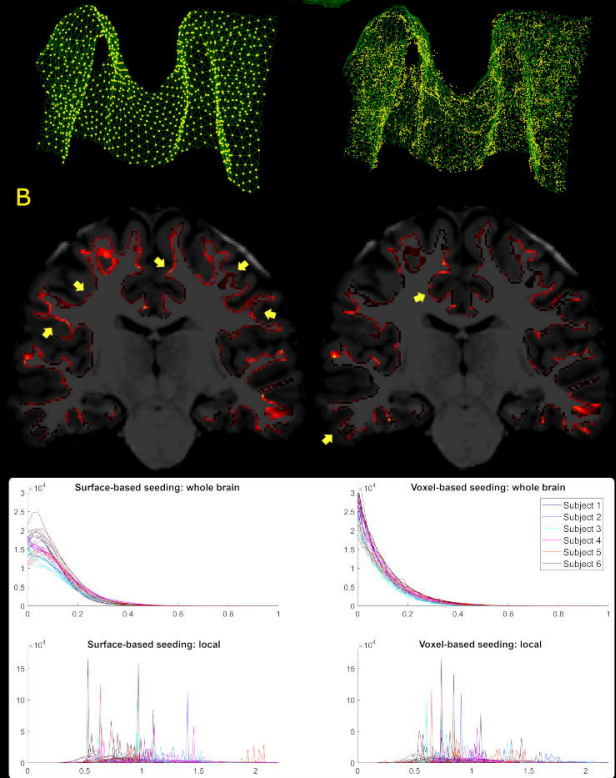


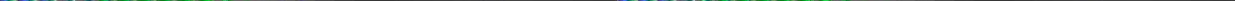




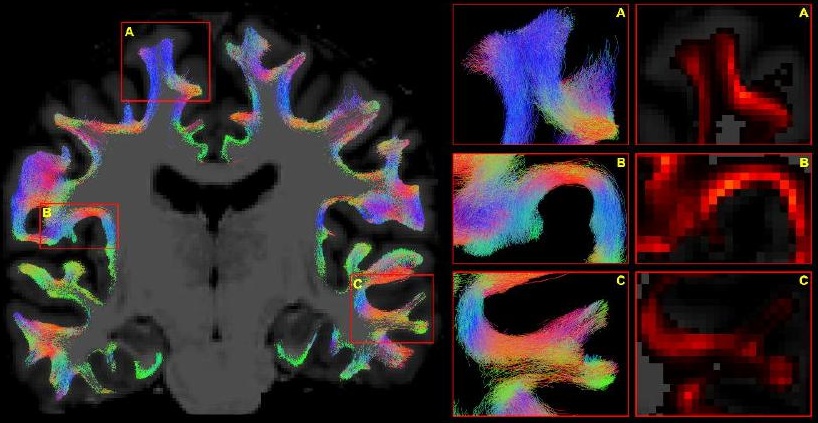


$\mathrm{CV}_{w}$

$x^{2}=-2 x$ ,

.

$\mathrm{CV}_{\mathrm{a}}$

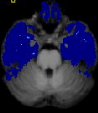

$\mathrm{ICC}$

in

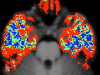

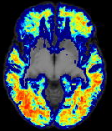
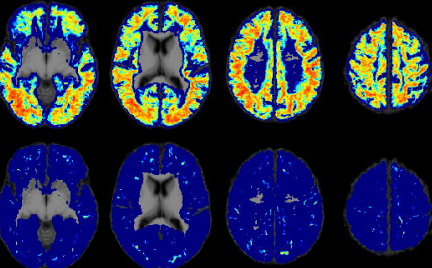

Aist
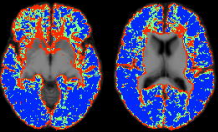

缕
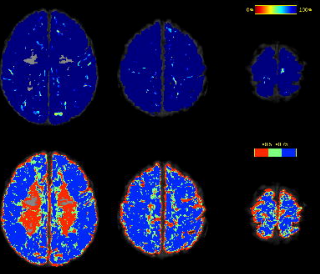

ates ot on
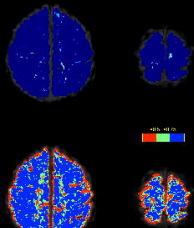


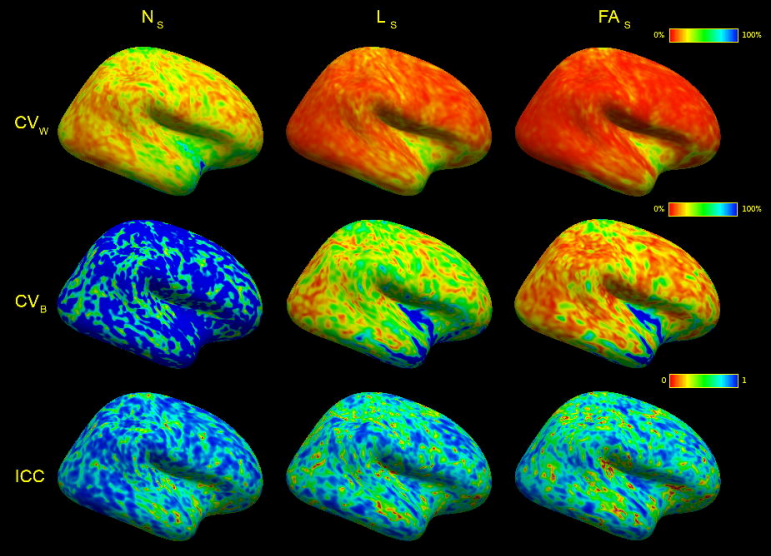

